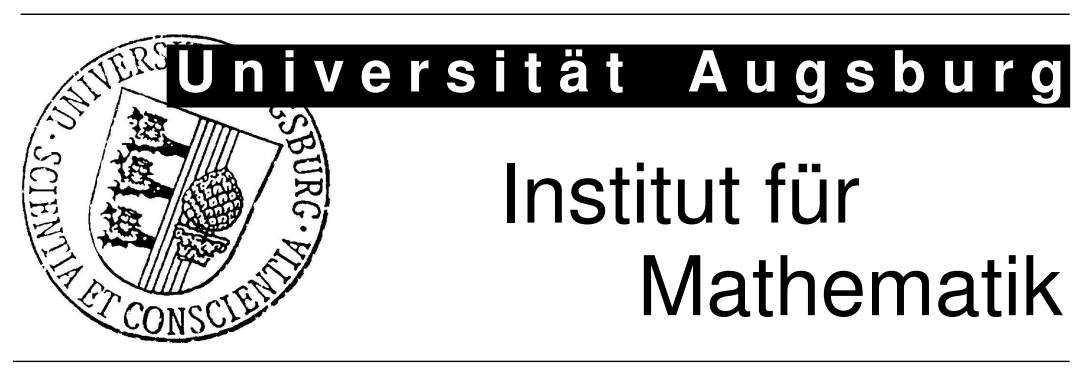

Kai-Friederike Oelbermann

Alternate Scaling Algorithm for Biproportional Divisor Methods

Preprint Nr. 04/2013 - 12. März 2013

Institut für Mathematik, Universitätsstraße, D-86135 Augsburg

http://www.math.uni-augsburg.de/ 


\section{Impressum:}

\section{Herausgeber:}

Institut für Mathematik

Universität Augsburg

86135 Augsburg

http://www . math. uni-augsburg.de/de/forschung/preprints.html

\section{ViSdP:}

Kai-Friederike Oelbermann

Institut für Mathematik

Universität Augsburg

86135 Augsburg

Preprint: Sämtliche Rechte verbleiben den Autoren (C) 2013 


\title{
Alternate Scaling algorithm for biproportional divisor methods
}

\author{
Kai-Friederike Oelbermann*
}

March 12, 2013

\begin{abstract}
In parliamentary elections biproportional divisor methods translate votes into seats so that, for each district, fixed seat contingents are met and that every party receives as many seats as the overall vote counts reflect. A set of district-divisors and party-divisors ensures that proportionality is respected both within the districts and within the parties. The divisors can be calculated by means of the alternating scaling algorithm (AS-algorithm) which is formally introduced. It is the discrete variant of the iterative proportional fitting procedure (IPF-procedure). The AS-algorithm iteratively generates scaled vote matrices that after rounding alternately fulfill the district-contingents and the party-seats. Thus it defines two sequences: the AS-seat-sequence and the AS-scaling-sequence. The central question in this paper is under which condition the AS-algorithm is able to generate the set of biproportional apportionments. The conjecture of Balinski \& Pukelsheim (2006) is proven stating that the AS-algorithm is effective for all biproportional apportionment problems that come with at most a few ties. In the rare event that the set of biproportional apportionments cannot be determined by the AS-algorithm, the complementary AS-Tie\&Transfer-combination puts things right. Its analysis leads to a constructive proof of necessary and sufficient conditions for the existence of biproportional apportionments. If these conditions are violated, the sequences generated by the AS-algorithms may have more than two accumulation points. On the contrary, the IPF-procedure has at most two accumulation points.
\end{abstract}

Keywords: alternate scaling algorithm, biproportional representation, biproportional electoral system, divisor methods, iterative proportional fitting, controlled rounding.

\section{Introduction and main results}

In numerous parliamentary elections the electoral area is subdivided into several districts. For example the election to the European Parliament takes place in 27 Member States and in German federal elections seats are allocated to parties that campaign in 16 Länder. A biproportional electoral system, first introduced by Balinski \& Demange (1989a,b) and Gassner (1989, 1991), secures proportionality with respect to the population figures of each district as well as the parties' total vote counts. It had its world premiere in 2006 during the Zurich municipal election (Pukelsheim \& Schuhmacher, 2004). Thereafter biproportional systems were applied during the municipal elections in Schaffhausen 2008, Aarau 2009, and Zurich 2010, and cantonal elections in Zurich 2007 and 2011, Schaffhausen 2009 and Aargau 2009 (Pukelsheim \& Schuhmacher, 2011). As an example table 2 displays vote counts and the resulting biproportional apportionment for the Zurich cantonal election in 2011.

In Switzerland biproportional apportionments are determined by the algorithm of alternating scaling (AS-algorithm). The AS-algorithm is a procedure for scaling rows and columns of an input $(k \times \ell)$-vote-matrix $V=\left(v_{i j}\right), v_{i j} \geq 0$, so that the output biproportional apportionment $B=\left(b_{i j}\right), b_{i j} \in \mathbb{N}_{0}$ achieves row sums equal to a pre-specified vector of district-contingents $r=\left(r_{1}, \ldots, r_{k}\right) \in \mathbb{N}^{k}$, and column sums equal to a pre-specified vector of party-seats $s=$

\footnotetext{
*Institute for Mathematics, Augsburg University, Oelbermann@math.uni-augsburg.de
} 
$\left(s_{1}, \ldots, s_{\ell}\right) \in \mathbb{N}^{\ell}$. District-contingents are generally determined in proportion to the districts' population figures. Party-seats are generally determined in proportion to the votes cast across the entire electoral area. The AS-algorithm performs what proportionality is about: Scale and round! Scaling within electoral districts achieves proportionality among the parties campaigning in that district. Scaling within parties secures parties to be handled proportionally across all districts. A final rounding step is inevitable, as deputies come in whole numbers.

The AS-algorithm iteratively calculates the AS-scaling-sequence $V(t)=\left(v_{i j}(t)\right), t=1,2$, etc., which emerges from the vote-matrix $V$ by scaling rows and columns. Furthermore, the ASalgorithm generates the $A S$-seat-sequence $\mathcal{A}(t)=\left\{A=\left(a_{i j}\right) \subset \mathbb{N}_{0}^{k \times \ell} \mid a_{i j} \in \llbracket v_{i j}(t) \rrbracket\right\}, t=1,2$, etc., where $\llbracket \cdot \rrbracket$ is a pre-specified rounding-rule. To make sure that the AS-seat-sequence fulfills the district-contingents in odd steps $2 t-1$ and complies with the party-seats in even steps $2 t$ the AS-scaling-sequence has to be chosen carefully. If the AS-seat-sequence converges then the set of biproportional apportionments $\mathcal{B}(V, r, s)$ is determined, there exists a time $t$ such that $\mathcal{A}(t)=\mathcal{B}(V, r, s)$. Otherwise there exists at least two accumulation points. In practice the set of biproportional apportionments is unique. However, ties are not impossible. If that should happen the election supervisor draws lots or other-sometimes very peculiar-rules apply. From a mathematical point of view ties are also very interesting. Amongst others they cause the rare events in which the AS-algorithm fails to determine the set of biproportional apportionments.

We study the AS-algorithm's convergence via an $L_{1}$-approach, as do Balinski \& Demange (1989b). To this end we measure the progress of the AS-seat-sequence via the $L_{1}$-error

$$
f(t):=f(A):=\sum_{i \leq k}\left|a_{i+}-r_{i}\right|+\sum_{j \leq \ell}\left|a_{+j}-s_{j}\right|
$$

for any apportionment $A=\left(a_{i j}\right) \in \mathcal{A}(t)$, where $a_{i+}$ and $a_{+j}$ indicate, respectively, the sum of entries in row $i$, and column $j$. The $L_{1}$-error is suggestive in that it counts along rows and columns how many seats are not yet adjusted. Usually the AS-algorithm determines exactly one apportionment in each step. Due to possible ties within the district or party adjustments multiple solutions may arise. Even more, their $L_{1}$-errors may vary. Therefore we define the AS-seat-sequence so that it comprises only the apportionments that minimize the $L_{1}$-error. The first versions of the Augsburg free-software BAZI (2013) had flaws in the minimizing procedure, such that data as in example 1 can only be handled since version 2012.07. Due to the minimizing step the AS-seat-sequence's $L_{1}$-error is well-defined and monotonically decreasing. Moreover it is bounded from below by the $L_{1}$-minimal-error $\lambda_{\min } . L_{1}$-minimal-error vanishes if and only if a biproportional apportionment exists. Existence, in turn, is checked via the flow-criterion (proposition 4). Consequently the $L_{1}$-error converges to the AS-limit-error $\lambda_{\mathrm{AS}}$ (proposition 5). The $L_{1}$-minimal-error and the AS-limit-error are essential for the ASalgorithm's analysis. In case the two figures coincide, $\lambda_{\min }=\lambda_{\mathrm{AS}}$, the AS-algorithm is called effective. Otherwise the AS-algorithm is called ineffective and the AS-limit-error is bounded from above by the ineffectiveness-error $\Lambda_{\text {ineff }}^{\mathrm{k}, \ell} \geq \lambda_{\mathrm{AS}}>\lambda_{\min }$ (proposition 6). Summing up, our analysis concentrates on three different error terms:

(1) $L_{1}$-minimal-error $\lambda_{\min }$ : The $L_{1}$-error of the AS-seat-sequence is bounded from below by the $L_{1}$-minimal-error, for all $t>1$ we get $f(t) \geq \lambda_{\min }$.

(2) AS-limit-error $\lambda_{\mathrm{AS}}$ : The $L_{1}$-error converges to the AS-limit-error, there exists a time $t^{\prime}$ such that for all $t \geq t^{\prime}$ we have $f(t)=f(t+1)=\lambda_{\mathrm{AS}} \geq \lambda_{\min }$.

(3) Ineffectiveness-error $\Lambda_{\text {ineff }}^{\mathrm{k}, \ell}$ : In case the AS-algorithm is ineffective, the AS-limit-error is bounded from above by the ineffectiveness-error, if $\lambda_{\mathrm{AS}}>\lambda_{\min }$ then $\Lambda_{\text {ineff }}^{\mathrm{k}, \ell} \geq \lambda_{\mathrm{AS}}$.

Theorem 1 shows that the AS-algorithm is always effective, if the set of biproportional apportionments comprises at most two ties, or if $k \cdot \ell<24$ holds for the number of districts and 
the number parties. As ties are rare the AS-algorithm works fine for all practical purposes. Thus theorem 1 substantiates the conjecture of Balinski \& Pukelsheim (2006). In case the flow-criterion is violated the AS-seat-sequence $\mathcal{A}(t)$ has two or more accumulation points (theorem 2). This result is somehow astonishing as the IPF-procedure-the continuous analogon which gets along without rounding-has at most two accumulation points. Concerning the ASscaling-sequence $V(t)$ theorem 3 states that convergence is on hand in case of ineffectiveness. Otherwise $V(t)$ may converge or diverge. Examples of the different scenarios are included in section 4. A juxtaposition of the (discrete) AS-algorithm and the (continuous) IPF-procedure is included in table 1.

\begin{tabular}{|c|c|c|c|}
\hline $\begin{array}{l}{ }^{a} \text { Effectivity/ } \\
{ }^{b} \text { Flow-criterion }\end{array}$ & $\begin{array}{l}{ }^{c} L_{1} \text {-minimal-error } /{ }^{d} \mathrm{AS}- \\
\text { boundary } /{ }^{e} \text { Ineffectiveness }\end{array}$ & ${ }^{f}$ AS-seat-sequence $\mathcal{A}(t)$ & $\begin{array}{c}{ }^{g} \text { Scaling-sequence } \\
V(t)\end{array}$ \\
\hline $\begin{array}{l}\text { AS-algorithm eff } \\
\text { fulfilled } \\
\text { violated } \\
\end{array}$ & $\begin{array}{ll}\text { ive: } & \\
\lambda_{\mathrm{AS}}=\lambda_{\min }=0 \\
\lambda_{\mathrm{AS}}=\lambda_{\min }>0\end{array}$ & $\begin{array}{l}\text { convergent } \\
\geq 2 \text { accumulation pts. }\end{array}$ & $\begin{array}{l}\text { convergent } \\
\text { div. or conv. }\end{array}$ \\
\hline $\begin{array}{l}\text { AS-algorithm in } \\
\text { fulfilled } \\
\text { violated }\end{array}$ & $\begin{array}{l}\text { ective: } \\
\quad \Lambda_{\text {ineff }}^{\mathrm{k}, \ell} \geq \lambda_{\mathrm{AS}}>\lambda_{\min }=0 \\
\Lambda_{\text {ineff }}^{\mathrm{k}, \ell} \geq \lambda_{\mathrm{AS}}>\lambda_{\min }>0\end{array}$ & $\begin{array}{l}2 \text { accumulation points } \\
2 \text { accumulation points }\end{array}$ & $\begin{array}{l}\text { convergent } \\
\text { convergent }\end{array}$ \\
\hline $\begin{array}{l}\text { IPF-procedure: } \\
\text { fulfilled } \\
\text { violated }\end{array}$ & & & $\begin{array}{c}\text { convergent } \\
2 \text { accumulation pts. }\end{array}$ \\
\hline
\end{tabular}

Table 1: AS-algorithm. Effectivity, that is $\lambda_{\mathrm{AS}}=\lambda_{\min }$ : If the flow-criterion is fulfilled, then the AS-seat-sequence determines the set of biproportional apportionments and the AS-scaling-sequence converges. Otherwise the AS-seat-sequence has at least two accumulation points and the AS-scalingsequence either converges or diverges. Ineffectivity, that is $\lambda_{\mathrm{AS}}>\lambda_{\min }$ : Independent from the flowcriterion the AS-limit-error is bounded from above by the ineffectiveness-error, the AS-seat-sequence has two accumulation points and the AS-scaling-sequence converges. IPF-procedure: The flow-criterion is fulfilled, if and only if the IPF-sequence converges. Otherwise it has two accumulation points.

\subsection{Literature survey}

An apportionment method is a mathematical provision to translate vote counts into seat numbers. On the basis of the apportionment problem in the American House of Representatives the monograph by Balinski \& Young (2001) elucidates different methods that were in use throughout history. Furthermore it discusses their mathematical properties. The difficulty in the translation of votes is the determination of integer seat numbers that are proportional to the votes and that sum up to a given house size. For divisor methods the votes are divided by a common divisor and the resulting quotients are rounded by a pre-specified rounding rule. In order to comply with the given house size, the divisor has to be chosen carefully.

If an electoral area is subdivided into several districts, a monoproportional apportionment method does not suffice. If fixed seat contingents are prescribed for the districts, a natural claim is to secure proportionality within both, the districts and the parties. To this end a biproportional divisor method secures a two-way proportionality. Votes are divided by partyspecific and district-specific divisors. The resulting quotients are rounded by a pre-specified rounding rule. In order to fulfill both, district-contingents and party-seats, the divisors have to be chosen carefully. To determine a feasible set of divisors Balinski \& Demange (1989b) introduce the Tie\&Transfer-algorithm. Its analysis leads to the flow-criterion-a necessary and sufficient condition for the existence of biproportional apportionments. The flow-criterion is violated if the total number of seats of some set of parties exceeds the number of seats that are rewarded to the districts in which these parties campaign. 
Balinski \& Pukelsheim (2006) propagate the algorithm of alternate scaling (AS-algorithm). Due to its intuitive line of action of alternately fitting district-contingents and party-seats the AS-algorithm is more attractive than the Tie\&Transfer-algorithm. However, Gaffke \& Pukelsheim (2008b) work out special problems for which the AS-algorithm fails to determine the set of biproportional apportionments. Balinski \& Pukelsheim (2006) conjecture that these pathological instances only appear in case of especially complicated ties. Maier (2008) and Maier et al. (2010) discuss the interplay between the AS-algorithm and the Tie\&Transferalgorithm. Intensive simulations suggest that the hybrid algorithm, which starts with alternate scaling and then switches to the Tie\&Transfer-algorithm, is faster than the simple algorithms. However, Maier (2008, page 49) ignores multiple solutions in his definition. Consequently his $L_{1}$-error is not well-defined and its monotonicity is not sufficiently backed up.

The AS-algorithm is the discrete analogon of the IPF-procedure. The IPF-procedure was popularized by Deming \& Stephan (1940). It may be applied in statistics to fit contingency-tables to pre-specified marginals. In the continuous variant the entries of the output biproportional fit $B=\left(b_{i j}\right)$ are nonnegative real numbers, $b_{i j} \in[0, \infty)$. It is well known that the IPF-sequence converges if and only if a biproportional fit exists (Bregman, 1967; Csiszár, 1975). If a biproportional fit does not exist, then the IPF-sequence has exactly two accumulation-points (Gietl \& Reffel, 2012). A biproportional fit is generally displayed in (rounded!) decimals. In order not to violate the given marginals Cox \& Ernst (1982) (see also Gassner $(1989,1991)$ in connection with electoral systems) discuss controlled roundings. However, the resulting apportionments are rarely comprehensible, verifiable and involve a complicated algorithm.

In contrast, the AS-algorithm permits a calculation of district-divisors and party-divisors. Once suitable divisors are publicized the outcome may be easily double-checked. One only needs to take the input weight of any cell, divide its row divisor and its column divisor, and round the quotient according to the pre-specified rounding rule. Thus-and despite rare cases of ineffectivity - we also advocate its application in statistics. BAZI (2013) is an open-software that computes biproportional apportionments. The user may choose to run the AS-algorithm, the Tie\&Transfer algorithm, or hybrid combinations of the two, as well as many other algorithms.

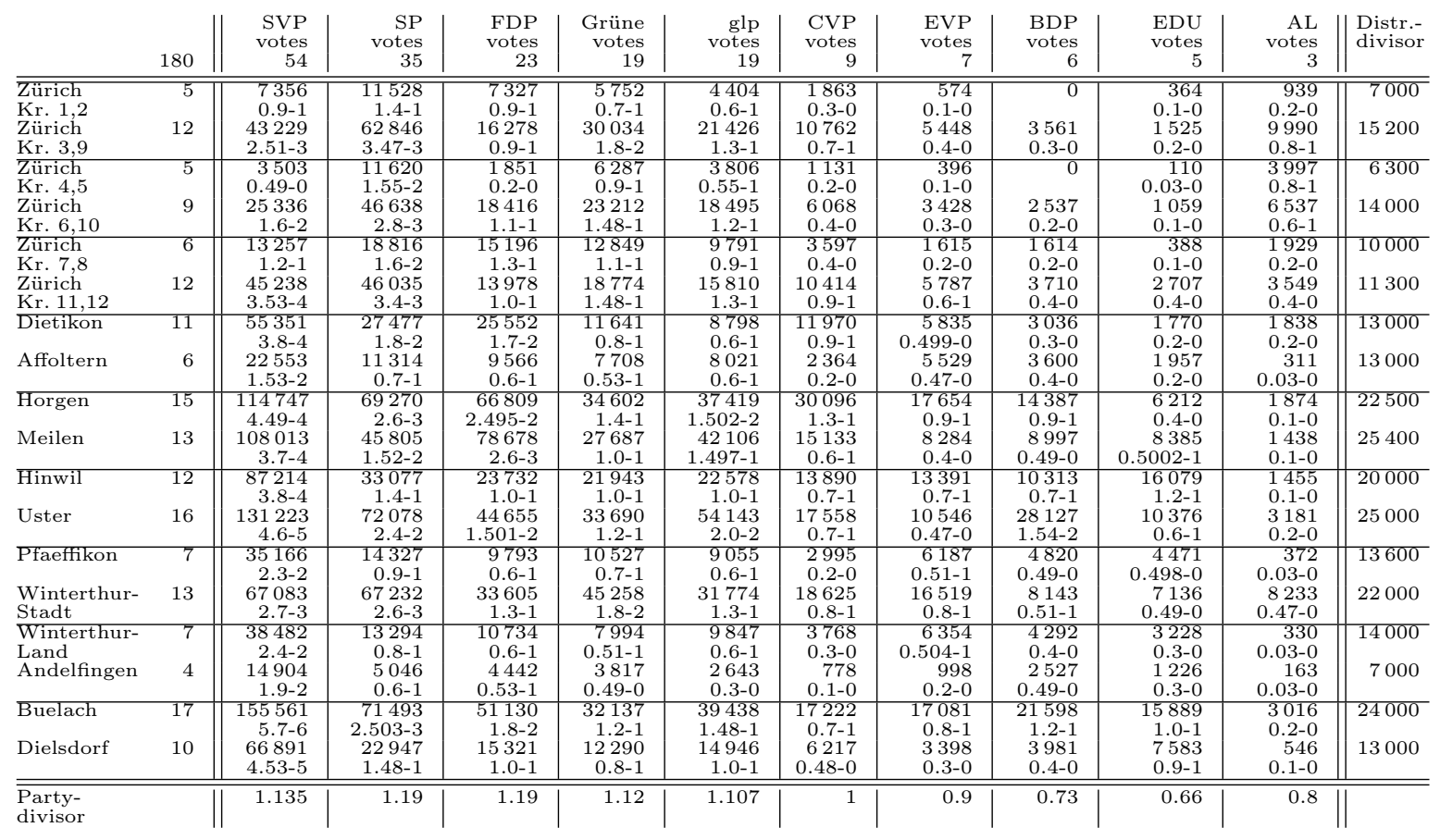

Table 2: Cantonal elections Zurich 2011. Votes are divided its district- and party-divisors. Decimals below .5 are rounded downwards, decimals above .5 are rounded upwards. The resulting integers display the biproportional apportionment. For example the SVP gained 7356 votes in the district of Zurich Kreis $1+2$. Divided by the respective divisors the quotient is $7356 /(7000 \cdot 1.135)=0.9$. Standard rounding yields one seat. The set of feasible divisors is determined by the AS-algorithm after 72 steps (variant 'midpoint'). Source: Pukelsheim \& Schuhmacher (2011). 


\section{Divisor methods}

Divisor methods determine apportionments, i.e. integer vectors or matrices with entries obtained by rounding scaled votes. We shall first introduce the notion of rounding rules as defined by Gaffke \& Pukelsheim (2008a). Rounding rules come with a one-to-one correspondence with signpost sequences. These designate dividing points below which quotients are rounded downwards and above which quotients are rounded upwards. By definition, a signpost sequence $s(1), s(2), \ldots$ equips each integer interval $[n-1, n]$ with a signpost $s(n) \in[n-1, n]$, with the sole restriction that there is no pair of two subscripts $\left(n, n^{\prime}\right) \in \mathbb{N} \times \mathbb{N}$ with $s(n)=n$ and $s\left(n^{\prime}\right)=n^{\prime}-1$. A signpost sequence defines a rounding rule $\llbracket \cdot \rrbracket$ from the half-line $[0, \infty)$ into oneor two-element integer subsets, through $\llbracket 0 \rrbracket:=\{0\}$ and $\llbracket v \rrbracket:=\left\{b \in \mathbb{N}_{0} \mid s(b) \leq v \leq s(b+1)\right\}$ for all $v>0$. For $v>0$ the definition may be restated in terms of the basic-relation

$$
b \in \llbracket v \rrbracket \Longleftrightarrow s(b) \leq v \leq s(b+1) .
$$

Rounding rules are monotonic in that $0<v^{\prime}<v$ implies $b^{\prime} \leq b$ for all $b^{\prime} \in \llbracket v^{\prime} \rrbracket$ and $b \in \llbracket v \rrbracket$. The boundary value $v=0$ is special as it is always rounded to the singleton $\{0\}$. For positive arguments $v>0$, a rounding rule returns the singleton $\{b\}$ as long as $v$ stays away from its neighboring signposts, $s(b)<v<s(b+1)$ implies $\llbracket v \rrbracket=\{b\}$. Ties of the sort $v=s(b)>0$ attract more attention. In such cases $v$ lies in two intervals, $[s(b-1), s(b)]$ and $[s(b), s(b+1)]$, such that two options are at hand: $v$ may be rounded downwards to $b-1$, or rounded upwards to $b$. Thus, the rounding rule returns a two-element set, $\llbracket v \rrbracket=\{b-1, b\}$.

A rounding rule is called pervious when the first signpost is positive, $s(1)>0$, and impervious when the first signpost is zero, $s(1)=0$. A pervious rounding rule maps arguments below $s(1)>0$ to zero, $v<s(1)$ implies $\llbracket v \rrbracket=\{0\}$. An impervious rounding rule yields zero if and only if the input is zero: $v=0$ if and only if $\llbracket v \rrbracket=\{0\}$.

For practical purpose rounding rules are often defined by stationary signpost sequences, $s_{r}(n):=n-1+r$ for $r \in[0,1]$. The signpost sequence $s_{1}(n):=n$ defines rounding downwards $\| \cdot \Downarrow$, the signpost sequence $s_{0.5}(n):=n-1 / 2$ yields standard rounding $\| \cdot \Downarrow$, and $s_{0}(n):=n-1$ specifies rounding upwards $\pi \cdot \pi$. For example we get

$$
\begin{aligned}
& \llbracket 1.5 \rrbracket=1 \quad\langle 1.5\rangle=\{1,2\} \quad \| 1.5 \rrbracket=2 \\
& \llbracket 2 \rrbracket=\{1,2\} \quad\langle 2\rangle=2 \quad \pi 2 \rrbracket=\{2,3\} .
\end{aligned}
$$

\subsection{Monoproportional apportionments}

Let $(v, h, \llbracket \cdot \rrbracket)$ define a monoproportional apportionment problem, where $v=\left(v_{1}, \ldots, v_{\ell}\right)$ is a positive vote-vector, $h$ denotes an integer house-size, and $\llbracket \cdot \rrbracket$ defines a rounding rule.

Definition 1 (Monoproportional apportionment). An integer vector $b=\left(b_{1}, \ldots, b_{\ell}\right) \in \mathbb{N}_{0}^{\ell}$ is called monoproportional apportionment (based on vote-vector $v$ and house-size $h$ ) when there exists a positive divisor $D$, such that the entries $b_{j}$ are obtained by scaling and rounding, $b_{j} \in \llbracket v_{j} / D \rrbracket$ for all parties $j \leq \ell$, and fit the house-size, $b_{+}:=b_{1}+\cdots+b_{\ell}=h$. The set $\mathfrak{b}(v, h)$ is defined to include all monoproportional apportionments. A positive divisor $D$ is called feasible when there exists a monoproportional apportionment $b=\left(b_{j}\right) \in \mathfrak{b}(v, h)$ so that $b_{j} \in \llbracket v_{j} / D \rrbracket$ for all parties $j \leq \ell$.

Let $b=\left(b_{j}\right) \in \mathfrak{b}(v, h)$ be a monoproportional apportionment. The basic-relation (1) translates to the min-max inequality

$$
\max _{j \leq \ell} \frac{s\left(b_{j}\right)}{v_{j}} \leq \min _{j \leq \ell} \frac{s\left(b_{j}+1\right)}{v_{j}}
$$


With the convention $v_{j} / 0:=\infty$ all divisors taken from the divisor-intervall

$$
\left[\max _{j \leq \ell} \frac{v_{j}}{s\left(b_{j}+1\right)}, \min _{j \leq \ell} \frac{v_{j}}{s\left(b_{j}\right)}\right]
$$

result in the same set of monoproportional apportionments. In the rare case that the divisorintervall is degenerated to a singleton, there exist two parties $j_{1} \neq j_{2}$ with $D=\frac{v_{j_{1}}}{s\left(b_{j_{1}}+1\right)}=\frac{v_{j_{2}}}{s\left(b_{j_{2}}\right)}$. For the quotients $v_{j_{1}} / D$ and $v_{j_{2}} / D$ the rounding rule is ambiguous,

$$
\llbracket \frac{v_{j_{1}}}{D} \rrbracket=\llbracket s\left(b_{j_{1}}+1\right) \rrbracket=\left\{b_{j_{1}}, b_{j_{1}}+1\right\} \quad \text { and } \quad \llbracket \frac{v_{j_{2}}}{D} \rrbracket=\llbracket s\left(b_{j_{2}}\right) \rrbracket=\left\{b_{j_{2}}-1, b_{j_{2}}\right\} .
$$

For party $j_{1}$ the quotient $v_{j_{1}} / D$ is rounded downwards and for party $j_{2}$ the quotient $v_{j_{2}} / D$ is rounded upwards. Pari passu the quotient $v_{j_{1}} / D$ may be rounded upwards and the quotient $v_{j_{2}} / D$ may be rounded downwards. Hence $b^{\prime}$, defined by $b_{j_{1}}^{\prime}:=b_{j_{1}}+1, b_{j_{2}}^{\prime}:=b_{j_{2}}-1$ and $b_{j}^{\prime}:=b_{j}$ for $j \neq j_{1}, j_{2}$, is another monoproportional apportionment, $b \neq b^{\prime} \in \mathfrak{b}(v, h)$. In this case the apportionments $b$ and $b^{\prime}$ are called ties.

Proposition 1 (Uniqueness). If a monoproportional apportionment exists, then it is unique up to ties.

Proof. Assume that two monoproportional apportionments do not result from the same divisor, $b_{j} \in \llbracket v_{j} / D \rrbracket$ and $b_{j}^{\prime} \in \llbracket v_{j} / D^{\prime} \rrbracket$ for all $j \leq \ell$ and $D \neq D^{\prime}$. If $b \neq b^{\prime}$, then there exists a party $j_{1}$ with $b_{j_{1}}>b_{j_{1}}^{\prime}$. The rounding rule's monotonicity implies $D \leq D^{\prime}$. Since both apportionments fulfill the house-size $h$, there exists another party $j_{2}$ with $b_{j_{2}}<b_{j_{2}}^{\prime}$ and thus $D \geq D^{\prime}$. Hence we get $D=D^{\prime}$ which is a contradiction to our assumption.

Proposition 2 (House-criterion). In case the rounding-rule is pervious there always exists a monoproportional apportionment $b \in \mathfrak{b}(v, h)$. In case the rounding-rule is impervious a monoproportional apportionment $b \in \mathfrak{b}(v, h)$ exists if and only if $h \geq \ell$.

Proof. Let the rounding rule be impervious. For $h=1$ the divisor $D=\max _{j \leq \ell} v_{j} / s(1)$ is feasible. The case $h>1$ follows by induction. Let the rounding rule be impervious. For $h=\ell$ all divisors $D \geq \max _{j \leq \ell} v_{j} / s(2)$ are feasible. The case $h>\ell$ follows by induction.

\subsection{Biproportional apportionments}

Let $(V, r, s, \llbracket \cdot \rrbracket)$ define a biproportional apportionment problem, where $V=\left(v_{i j}\right)$ is a nonnegative $(k \times \ell)$-vote-matrix, $r=\left(r_{1}, \ldots, r_{k}\right) \in \mathbb{N}^{k}$ a vector of integer district-contingents, $s=\left(s_{1}, \ldots, s_{\ell}\right) \in \mathbb{N}^{\ell}$ a vector of integer party-seats, and $\llbracket \cdot \rrbracket$ a rounding rule. Without loss of generality we assume that $r_{+}=s_{+}$holds and that $V$ is connected, i.e. permutations of rows and columns do not allow a representation

$$
\begin{array}{c|cc} 
& \multicolumn{1}{c}{J} & J^{c} \\
\hline I & \left(\begin{array}{cc}
V^{(I \times J)} & 0 \\
0 & V^{\left(I^{c} \times J^{c}\right)}
\end{array}\right)
\end{array},
$$

where at least one of the subsets $I$ or $J$ is non-empty and proper.

Definition 2 (Biproportional apportionment). An integer matrix $B=\left(b_{i j}\right) \in \mathbb{N}_{0}^{k \times \ell}$ is called biproportional apportionment (based on vote-matrix $V$, district-contingents $r$ and party-seats $s$ ) when there exists a set of district-divisors $x=\left(x_{1}, \ldots, x_{k}\right)>0$ and party-divisors $y=$ $\left(y_{1}, \ldots, y_{\ell}\right)>0$ so that all entries $b_{i j}$ are obtained by scaling and rounding, $b_{i j} \in \llbracket v_{i j} /\left(x_{i} y_{j}\right) \rrbracket$, and fit the marginals, $b_{i+}:=b_{i 1}+\cdots+b_{i \ell}=r_{i}$ and $b_{+j}:=b_{1 j}+\cdots b_{k j}=s_{j}$ for all districts $i \leq k$ and for all parties $j \leq \ell$. The set $\mathcal{B}(V, r, s)$ is defined to include all biproportional apportionments. A set of divisors $(x, y)>0$ is called feasible when there exists a biproportional apportionment $B=\left(b_{i j}\right) \in \mathcal{B}(V, r, s)$ so that $b_{i j} \in \llbracket v_{i j} /\left(x_{i} y_{j}\right) \rrbracket$ for all $i \leq k$ and all $j \leq \ell$. 
Gaffke \& Pukelsheim (2008a, Theorem 7.1) show that any apportionment $A=\left(a_{i j}\right) \in \mathbb{N}_{0}^{k \times \ell}$ is a biproportional apportionment if and only if it fulfills the critical inequalities

$$
\prod_{p \leq q} \frac{s\left(a_{i_{p} j_{p}}\right)}{v_{i_{p} j_{p}}} \leq \prod_{p \leq q} \frac{s\left(a_{i_{p+1} j_{p}}+1\right)}{v_{i_{p+1} j_{p}}} \quad \forall\left(i_{(q)}, j_{(q)}\right) \subseteq \operatorname{supp}(V),
$$

where $\left(i_{(q)}, j_{(q)}\right):\left(i_{1}, j_{1}\right) \rightarrow\left(i_{2}, j_{1}\right) \rightarrow\left(i_{2}, j_{2}\right) \rightarrow \ldots \rightarrow\left(i_{q}, j_{q}\right) \rightarrow\left(i_{1}, j_{q}\right)$ is a cycle on $V$ so that $i_{p}, j_{p} \in \operatorname{supp}(V):=\left\{(i, j) \mid v_{i j} \neq 0\right\}$ and $i_{p} \notin\left\{i_{1}, \ldots, i_{p-1}\right\}, j_{p} \notin\left\{j_{1}, \ldots, j_{p-1}\right\}$ for all $p \leq q$.

In practice the set of biproportional apportionments is a singleton, $\mathcal{B}(V, r, s)=\left\{B=\left(b_{i j}\right)\right\}$. In this case the critical inequalities (4) are strict and a set of divisors $(x, y)$ can be chosen such that the rounding of the quotient $v_{i j} /\left(x_{i} y_{j}\right)$ is unique for all parties in all districts,

$$
s\left(b_{i j}\right)<\frac{v_{i j}}{x_{i} y_{j}}<s\left(b_{i j}+1\right) \quad \forall i \leq k, \forall j \leq \ell .
$$

In theorie, however, there might be a cycle $\left(i_{1}, j_{1}\right) \rightarrow\left(i_{2}, j_{1}\right) \rightarrow \ldots \rightarrow\left(i_{q}, j_{q}\right) \rightarrow\left(i_{1}, j_{q}\right)$ such that (4) holds with equality,

$$
\frac{v_{i_{p} j_{p}}}{x_{i_{p}} y_{j_{p}}}=s\left(b_{i_{p} j_{p}}\right) \quad \text { and } \quad \frac{v_{i_{p+1} j_{p}}}{x_{i_{p+1}} y_{j_{p}}}=s\left(b_{i_{p+1} j_{p}}+1\right) \quad \forall p \leq q
$$

For party $j_{p}$ in district $i_{p}$ the quotient $v_{i_{p} j_{p}} /\left(x_{i_{p}} y_{j_{p}}\right)$ is rounded upwards while in district $i_{p+1}$ the quotient $v_{i_{p+1} j_{p}} /\left(x_{i_{p+1} y_{p}}\right)$ is rounded downwards. Pari passu the quotient $v_{i_{p} j_{p}} /\left(x_{i_{p}} y_{j_{p}}\right)$ may be rounded downwards and the quotient $v_{i_{p+1} j_{p}} /\left(x_{i_{p+1} y_{p}}\right)$ may be rounded upwards. Hence $B^{\prime}=\left(b_{i j}^{\prime}\right)$, defined by

$$
b_{i j}^{\prime}:= \begin{cases}b_{i j}-1 & \text { if }(i, j)=\left(i_{p}, j_{p}\right) \text { for one } p \leq q, \\ b_{i j}+1 & \text { if }(i, j)=\left(i_{p+1}, j_{p}\right) \text { for one } p \leq q, \\ b_{i j} & \text { otherwise }\end{cases}
$$

is another biproportional apportionment, $B \neq B^{\prime} \in \mathcal{B}(V, r, s)$. In this case the apportionments $B$ and $B^{\prime}$ are called ties. Balinski \& Demange (1989a) and Gaffke \& Pukelsheim (2008a) show that biproportional apportionments are unique up to ties.

Proposition 3 (Uniqueness). If a biproportional apportionment exists, then it is unique up to ties.

Proof. Our proof relies on Pukelsheim's (2012) proof of his first theorem which states that a continuous biproportional fit is unique. Let $B=\left(b_{i j}\right)$ and $B^{\prime}=\left(b_{i j}^{\prime}\right) \in \mathcal{B}(V, r, s)$ be two biproportional apportionments with $b_{i j} \in \llbracket v_{i j} /\left(x_{i} y_{j}\right) \rrbracket$ and $b_{i j}^{\prime} \in \llbracket v_{i j} /\left(u_{i} v_{j}\right) \rrbracket$. Since marginal sums of $B$ and $B^{\prime}$ coincide, $r_{i}=b_{i+}=b_{i+}^{\prime}$ and $s_{j}=b_{+j}=b_{+j}^{\prime}$, unequal entries in some cells must be evened out through unequal entries in other cells. We construct a cycle on the support of the matrix $B-B^{\prime}$. As $v_{i j}=0$ implies $b_{i j}=b_{i j}^{\prime}=0$ all entries $v_{i j}$ along the cycle are positive. We start in a cell $\left(i_{1}, j_{1}\right)$ with $b_{i_{1} j_{1}}>b_{i_{1} j_{1}}^{\prime}$. The rounding rule's monotonicity implies $x_{i_{1}} y_{j_{1}} \leq u_{i_{1}} v_{j_{1}}$. For party $j_{1}$ there exists a district $i_{2}$ with $b_{i_{2} j_{1}}<b_{i_{2} j_{1}}^{\prime}$ and thus $x_{i_{2}} y_{j_{1}} \geq u_{i_{2}} v_{j_{1}}$. Furthermore for district $i_{2}$ there exists a party $j_{2}$ with $b_{i_{2} j_{2}}>b_{i_{2} j_{2}}^{\prime}$ and thus $x_{i_{2}} y_{j_{2}} \leq u_{i_{2}} v_{j_{2}}$, etc. The cycle closes in a cell $\left(i_{1}, j_{q}\right)$ with $b_{i_{1} j_{q}}<b_{i_{1} j_{q}}^{\prime}$ and $x_{i_{1}} y_{j_{q}} \geq u_{i_{1}} v_{j_{q}}$. We get

$$
\prod_{p \leq q} x_{i_{p}} y_{j_{p}} \leq \prod_{p \leq q} u_{i_{p}} v_{j_{p}}=\prod_{p \leq q} u_{i_{p+1}} v_{j_{p}} \leq \prod_{p \leq q} x_{i_{p+1}} y_{j_{p}}=\prod_{p \leq q} x_{i_{p}} y_{j_{p}} .
$$

Thus the sets of divisors coincide, $\prod_{p \leq q} x_{i_{p}} y_{j_{p}}=\prod_{p \leq q} u_{i_{p}} v_{j_{p}}$ for all $p \leq q$. By construction $b_{i_{p} j_{p}}^{\prime}=b_{i_{p} j_{p}}-1$ and $b_{i_{p+1} j_{p}}^{\prime}=b_{i_{p+1} j_{p}}+1$ holds. Hence, $B$ and $B^{\prime}$ are ties. 
Next we want to establish whether a biproportional apportionment exists. The following notations turn out to be helpful. The partial sum of entries $r_{i}$ over a subset $I \subseteq\{1, \ldots, k\}$ is denoted by $r_{I}:=\sum_{i \in I} r_{i}$. For vectors $s=\left(s_{1}, \ldots, s_{\ell}\right)$ and subsets $J \in\{1, \ldots, \ell\}$ the notation extends to $s_{J}:=\sum_{j \in J} s_{j}$. Sums over the empty set are taken to be zero, $r_{\emptyset}=s_{\emptyset}=0$. For keeping track of the nonzero entries in the vote-matrix $V$ we associate with every district subset $I \subseteq\{1, \ldots, k\}$ the set of parties connected in $V$ to $I, J_{V}(I):=\left\{j \leq \ell \mid v_{i j}>0\right.$ for some $\left.i \in I\right\}$. The complement $J_{V}(I)^{c}:=\{1, \ldots, \ell\} \backslash J_{V}(I)$ embraces parties $j$ with $v_{i j}=0$ in all districts $i \in I$. Hence the $\left(I \times J_{V}(I)^{c}\right)$-submatrix of $V$ vanishes and the sum of its entries is zero. The support-matrix of $V$ is defined to be

$$
E=\left(e_{i j}\right) \quad \text { with } \quad e_{i j}:= \begin{cases}1 & \text { if }(i, j) \in \operatorname{supp}(V) \\ 0 & \text { otherwise }\end{cases}
$$

Let the rounding rule be pervious. If there exists a subset $I \subseteq\{1, \ldots, k\}$ with $r_{I}>s_{J_{V}(I)}$, then the total number of seats to be allocated in districts $i \in I$ exceeds the number of seats that parties $j \in J_{V}(I)$ are entitled to. Hence no biproportional apportionment exists. This argument extends to the flow-criterion

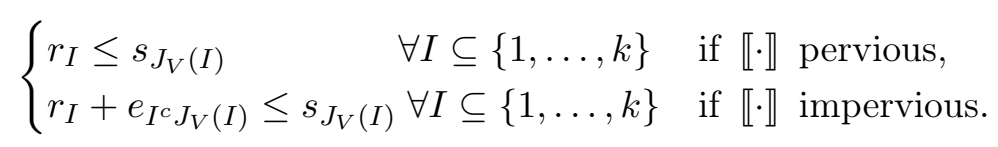

In connection with the theorie of networks the first inequality is often named after the American mathematicians David Gale (1921-2008) and Alan J. Hoffman $\left({ }^{*} 1924\right)$ (see Gale (1957) and Hoffman (1960)). Balinski \& Demange (1989b) show that the flow-criterion is necessary and sufficient for the existence of a biproportional apportionment.

Proposition 4 (Flow-criterion). Let $(V, r, s, \llbracket \cdot \rrbracket)$ be a biproportional apportionment problem. A biproportional apportionment exists if and only if the flow-criterion (6) is fulfilled.

Proof. Our proof in section 10 is based on the analysis of the AS-algorithm, whereas Balinski and Demange's proof is based on their Tie\&Transfer-algorithm.

\section{Formalization of the AS-algorithm}

The AS-algorithm is initialized by those apportionments $A \in \mathbb{N}_{0}^{k \times \ell}$ that are obtained from the vote-matrix $V$ by rounding and that minimize the $L_{1}$-error,

$$
\mathcal{A}(0):=\left\{A=\left(a_{i j}\right) \mid a_{i j} \in \llbracket v_{i j} \rrbracket\right\} .
$$

In its first step the AS-algorithm defines $\rho_{i}(1):=1$ for all districts $i \in I^{=}(0):=\left\{i \leq k \mid a_{i+}=\right.$ $r_{i}$ for all $\left.A \in \mathcal{A}(0)\right\}$. For the remaining districts $i \in I^{=}(0)^{c}$ the AS-algorithm solves the monoproportional apportionment problem that arises from the district votes $v_{i}(0):=\left(v_{i 1}, \ldots, v_{i \ell}\right)$ and the district-contingent $r_{i}$. To this end a feasible district-divisor $\rho_{i}(1)$ is determined, such that the sum of the rounded quotients fulfills the district-contingent. We define $V(1)=\left(v_{i j}(1)\right)$ by $v_{i j}(1):=v_{i j} / \rho_{i}(1)$. Subsequently we identify those apportionments $A$ that are obtained from $V(1)$ by rounding, that fulfill all district-contingents and that minimize the $L_{1}$-error $f(A):=\sum_{i \leq k}\left|a_{i+}-r_{i}\right|+\sum_{j \leq \ell}\left|a_{+j}-s_{j}\right|$. That is, we define

$$
\mathcal{A}(1):=\left\{A=\left(a_{i j}\right) \mid a_{i j} \in \llbracket v_{i j}(1) \rrbracket, a_{i+}=r_{i}, f(A)=\min _{\substack{B=\left(b_{i j}\right), b_{i j} \in \llbracket v_{i j}(1) \rrbracket \\ b_{i+}=r_{i}}} f(B)\right\} .
$$


In practice the roundings are unique. Accordingly the minimization is dispensable and the set of apportionments is unique. In theorie, however, the minimization is essential. The appendant algorithm is part of the Tie\&Transfer-algorithm (Balinski \& Demange, 1989b).

If the $L_{1}$-error of $A \in \mathcal{A}(1)$ vanishes, then the set of biproportional apportionments is determined. However, generally this is not the case. Some parties may have too many seats while others have too little.

In the second step the AS-algorithm defines $\sigma_{j}(2):=1$ for all parties $j \in J^{=}(1):=\{j \leq$ $\ell \mid a_{+j}=s_{j}$ for all $\left.A \in \mathcal{A}(1)\right\}$. For parties $j \in J^{=}(1)^{c}$ the AS-algorithm solves the monoproportional apportionment problem that arises from the scaled party-votes $v_{j}(1):=\left(v_{1 j}(1), \ldots, v_{k j}(1)\right)$ and the party-seats $s_{j}$. To this end a feasible party-divisor $\sigma_{j}(2)$ is determined. We define $V(2)=\left(v_{i j}(2)\right)$ by $v_{i j}(2):=v_{i j}(1) / \sigma_{j}(2)$ and identify those apportionments that minimize the $L_{1}$-error,

$$
\mathcal{A}(2):=\left\{A=\left(a_{i j}\right) \mid a_{i j} \in \llbracket v_{i j}(2) \rrbracket, a_{+j}=s_{j}, f(A)=\min _{\substack{B=\left(b_{i j}\right), b_{i j} \in \llbracket v_{i j}(2) \rrbracket \\ b_{+j}=s_{j}}} f(B)\right\} .
$$

For a general step $t=1,2$, etc. the AS-algorithm generates incremental district-divisors $\rho_{i}(2 t-$ 1 ) for all districts $i \leq k$, and incremental party-divisors $\sigma_{j}(2 t)$ for all parties $j \leq \ell$. Those identify cumulative district-divisors and cumulative party-divisors

$$
x_{i}(2 t-1):=x_{i}(2 t):=\rho_{i}(1) \rho_{i}(3) \cdots \rho_{i}(2 t-1), \quad y_{j}(2 t):=y_{j}(2 t+1):=\sigma_{j}(2) \sigma_{j}(4) \cdots \sigma_{j}(2 t) .
$$

The cumulative divisors in turn determine the AS-scaling-sequence

$$
V(t)=\left(v_{i j}(t)\right):=\left(\frac{v_{i j}}{x_{i}(t) y_{j}(t)}\right)
$$

which is examined section 6. The AS-scaling-sequence generates the $A S$-seat-sequence $\mathcal{A}(t)$,

$$
\begin{gathered}
\mathcal{A}(2 t-1):=\left\{A=\left(a_{i j}\right) \mid a_{i j} \in \llbracket v_{i j}(2 t-1) \rrbracket, a_{i+}=r_{i}, f(A)=\min _{B=\left(b_{i j}\right), b_{i j} \in \llbracket v_{i j}(2 t-1) \rrbracket,} f(B)\right\}, \\
\mathcal{A}(2 t):=\left\{A=\left(a_{i j}\right) \mid a_{i j} \in \llbracket v_{i j}(2 t) \rrbracket, a_{+j}=s_{j}, f(A)=\min _{B=\left(b_{i j}\right), b_{i j} \in \llbracket v_{i j}(2 t) \rrbracket,} f(B)\right\}, \\
b_{+j}=s_{j}
\end{gathered}
$$

which is scrutinized in section 7 . The $L_{1}$-error of the $A S$-algorithm defined through

$$
f(t):=f(\mathcal{A}(t)):=f(A)=\sum_{i \leq k}\left|a_{i+}-r_{i}\right|+\sum_{j \leq \ell}\left|a_{+j}-s_{j}\right| \quad \text { for any } \quad A \in \mathcal{A}(t) .
$$

Note that $f(t)$ does not depend on a particular apportionment, because all apportionments $A \in \mathcal{A}(t)$ come with the same $L_{1}$-error. In section 5 we proof that $f(t)$ is monotonically decreasing.

For the analysis of the AS-algorithm the sets of over-represented, under-represented and matching districts are essential,

$$
I^{+}(t):=\bigcup_{A \in \mathcal{A}(t)} I^{+}(A), \quad I^{-}(t):=\bigcup_{A \in \mathcal{A}(t)} I^{-}(A), \quad I^{=}(t):=\bigcap_{A \in \mathcal{A}(t)} I^{=}(A),
$$

with $I^{+}(A):=\left\{i \leq k \mid a_{i+}>r_{i}\right\}, I^{-}(A):=\left\{i \leq k \mid a_{i+}<r_{i}\right\}$ and $I^{=}(A):=\left\{i \leq k \mid a_{i+}=r_{i}\right\}$. 
Analogously the sets of over-represented, under-represented and matching parties, are given by

$$
J^{+}(t):=\bigcup_{A \in \mathcal{A}(t)} J^{+}(A), \quad J^{-}(t):=\bigcup_{A \in \mathcal{A}(t)} J^{-}(A), \quad J^{=}(t):=\bigcap_{A \in \mathcal{A}(t)} J^{=}(A) .
$$

As district-contingents are adjusted in odd steps we have $I^{=}(2 t-1)=\{1, \ldots, k\}$. Analogously we get $J^{=}(2 t)=\{1, \ldots, \ell\}$. Recall that $\mathcal{A}(t)$ comprises only those apportionments that minimize the $L_{1}$-error. Hence the sets defined above are disjoint (Balinski \& Demange, 1989b). Without loss of generality let the house-criterion of proposition 2 hold for all monoproportional apportionment problems. Therewith the incremental divisors can be chosen from the divisorintervalls that result from the max-min inequality (2). Let $A \in \mathcal{A}(2 t-1)$ be any apportionment. The incremental district-divisors $\rho_{i}(2 t-1)$ satisfy

$$
\rho_{i}(2 t-1) \in\left\{\begin{array}{cl}
{\left[\max _{j \leq \ell} \frac{v_{i j}(2 t-2)}{s\left(a_{i j}+1\right)}, \min _{j \leq \ell} \frac{v_{i j}(2 t-2)}{s\left(a_{i j}\right)}\right]} & \text { if } i \in I^{=}(2 t-2)^{c}, \\
{[1,1]} & \text { if } i \in I^{=}(2 t-2) .
\end{array}\right.
$$

Let $A^{\prime} \in \mathcal{A}(2 t)$. The incremental party-divisors $\sigma_{j}(2 t)$ satisfy

$$
\sigma_{j}(2 t) \in\left\{\begin{array}{cl}
{\left[\max _{i \leq k} \frac{v_{i j}(2 t-1)}{s\left(a_{i j}^{\prime}+1\right)}, \min _{i \leq k} \frac{v_{i j}(2 t-1)}{s\left(a_{i j}^{\prime}\right)}\right]} & \text { if } j \in J^{=}(2 t-1)^{c}, \\
{[1,1]} & \text { if } j \in J^{=}(2 t-1) .
\end{array}\right.
$$

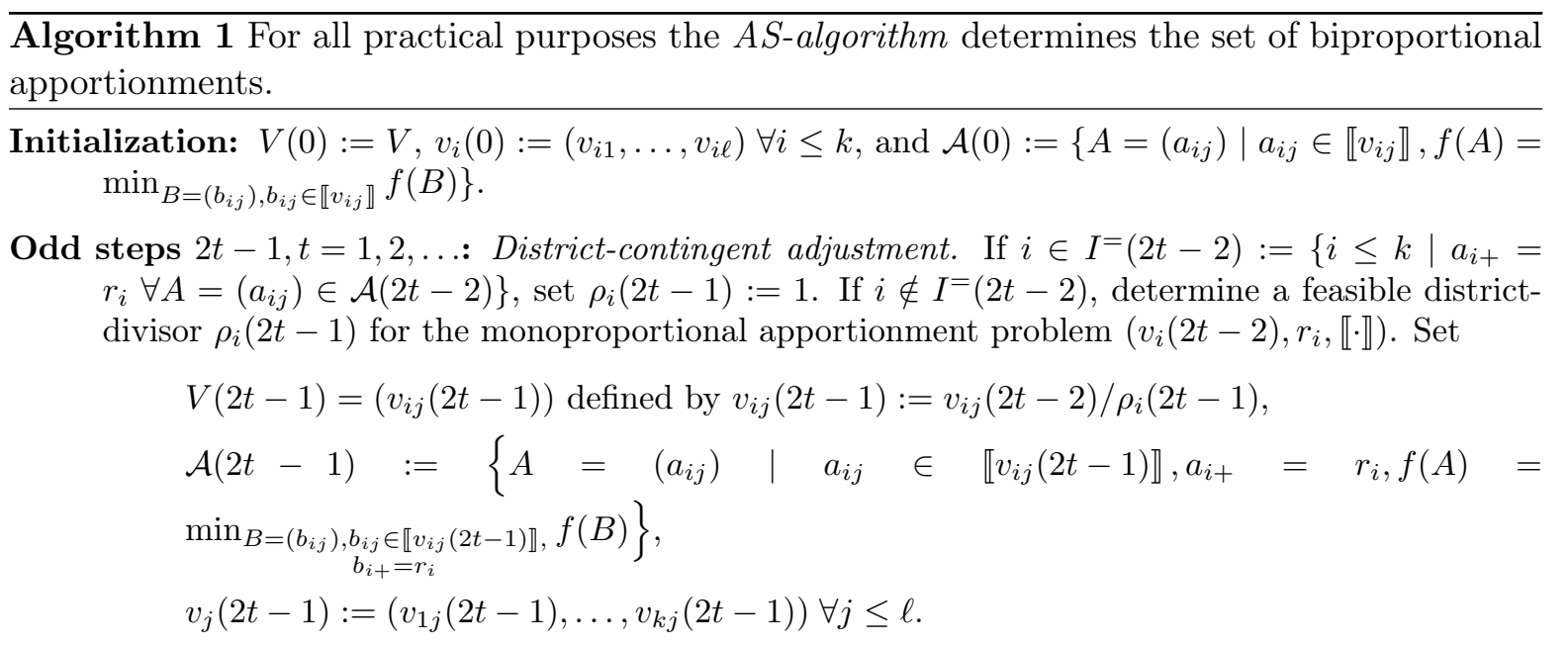

Even Steps 2t: Party-seat adjustment. If $j \in J^{=}(2 t-1):=\left\{j \leq \ell \mid a_{+j}=s_{j} \forall A=\left(a_{i j}\right) \in\right.$ $\mathcal{A}(2 t-1)\}$, set $\sigma_{j}(2 t):=1$. If $j \notin J=(2 t-1)$, determine a feasible party-divisor $\sigma_{j}(2 t)$ for the monoproportional apportionment problem $\left(v_{j}(2 t-1), s_{j}, \llbracket \cdot \rrbracket\right)$. Set

$$
\begin{aligned}
& V(2 t)=\left(v_{i j}(2 t)\right) \text { defined by } v_{i j}(2 t):=v_{i j}(2 t-1) / \sigma_{j}(2 t), \\
& \mathcal{A}(2 t):=\left\{A=\left(a_{i j}\right) \mid a_{i j} \in \llbracket v_{i j}(2 t) \rrbracket, a_{+j}=s_{j}, f(A)=\min _{\left.B=\left(b_{i j}\right), b_{i j} \in \llbracket v_{i j}(2 t) \rrbracket, f(B)\right\},} b_{+j}=s_{j}\right. \\
& v_{i}(2 t):=\left(v_{i 1}(2 t), \ldots, v_{i \ell}(2 t)\right) \forall i \leq k .
\end{aligned}
$$

Output: AS-seat-sequence $\mathcal{A}(t)$, AS-scaling-sequence $V(t)$, sequence of incremental divisors $\left(\rho_{i}(2 t-1)\right)_{i \leq k}$ and $\left(\sigma_{j}(2 t)\right)_{j \leq \ell}$, and sequence of cumulative divisors $\left(x_{i}(t)\right)_{i \leq k}$ and $\left(y_{j}(t)\right)_{j \leq \ell}$ for all $t=1,2$, etc. 


\section{Five examples}

The AS-algorithm is implemented in BAZI (2013). The max-min-equality (2) implies that the AS-scaling-sequence $V(t)$ and the AS-seat-sequence $\mathcal{A}(t)$ are not unambiguous, but depend on the incremental divisors taken from the respective divisor intervalls. Relating to the divisors' choice the BAZI-user may select one out of three variants. 'Midpoint' (mdpt): Divisors taken from the middle of each interval. 'Random' (rand): Uniformly distributed point in each interval. 'Extreme' (extr): Interval's lower bound for $i \in I^{-}(2 t-2)$ and $j \in J^{-}(2 t-1)$, and interval's upper bound for $i \in I^{+}(2 t-2)$ and $j \in J^{+}(2 t-1)$.

The variant 'weak' seems also feasible: Interval's upper bound for $i \in I^{-}(2 t-2)$ and $j \in$ $J^{-}(2 t-1)$, and interval's lower bound for $i \in I^{+}(2 t-2)$ and $j \in J^{+}(2 t-1)$. However, the upcoming example 1 reveals that it should be circumvented. The following five problems exemplify the AS-algorithm. We analyse biproportional problems with a vanishing AS-limiterror (example 1), with a positive AS-limit-error that coincides with the $L_{1}$-minimal-error (examples 2 and 3), and problems for which the AS-algorithm is ineffective (examples 4 and 5). The different variants of the AS-algorithm are labeled with upper indices; e.g. $\rho_{i}^{(\mathrm{mdpt})}(2 t-1)$, $\sigma_{j}^{(\mathrm{mdpt})}(2 t)$ for incremental divisors under variant 'midpoint'.

Example 1 (Effectivity). Let us study the following biproportional problem with standard rounding and vanishing $L_{1}$-minimal-error, $\lambda_{\min }=0$ :

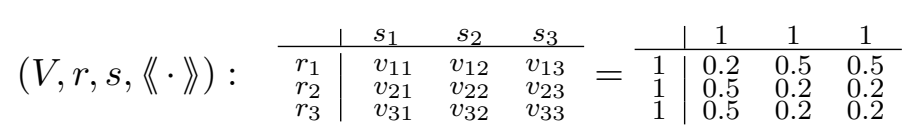

In the AS-algorithm's first step district-divisors are determined given the restrictions from equality $(7), \rho_{1}(1) \in[1,1], \rho_{2}(1) \in[0.4,1]$ and $\rho_{3}(1) \in[0.4,1]$. For district-divisors under variant 'weak' $\rho_{1}^{\text {(weak) }}(1)=\rho_{2}^{\text {(weak) }}(1)=\rho_{3}^{\text {(weak) }}(1)=1$, there exist two apportionments which comply with the district-contingents and minimize the $L_{1}$-error,

$$
V^{\text {(weak) }}(1)=\left(\begin{array}{ccc}
0.2 & 0.5 & 0.5 \\
0.5 & 0.2 & 0.2 \\
0.5 & 0.2 & 0.2
\end{array}\right), \quad \mathcal{A}^{\text {(weak) }}(1)=\left\{\left(\begin{array}{lll}
0 & 1 & 0 \\
1 & 0 & 0 \\
1 & 0 & 0
\end{array}\right),\left(\begin{array}{lll}
0 & 0 & 1 \\
1 & 0 & 0 \\
1 & 0 & 0
\end{array}\right)\right\}, \quad f^{(\text {weak })}(1)=2 .
$$

The only over-represented party is $J^{(\text {weak })+}(1)=\{1\}$. Under-represented parties are $J^{(\text {weak })-}(1)$ $=\{2,3\}$. In the AS-algorithm's second step the incremental party-divisors are determined given the restrictions from equation $(8), \sigma_{1}(2) \in[1,1], \sigma_{2}(2) \in[0.4,1]$ and $\sigma_{3}(2) \in[0.4,1]$. In case we continue with variant 'weak', then the AS-scaling-sequence is constant, $V=V^{(\text {weak })}(1)=$ $V^{(\text {weak })}(2)=\cdots$, and the AS-seat-sequence has two accumulation-points, comprising two apportionments each,

$$
\mathcal{A}^{\text {(weak) }}(2 t-1)=\left\{\left(\begin{array}{lll}
0 & 1 & 0 \\
1 & 0 & 0 \\
1 & 0 & 0
\end{array}\right),\left(\begin{array}{lll}
0 & 0 & 1 \\
1 & 0 & 0 \\
1 & 0 & 0
\end{array}\right)\right\}, \quad \mathcal{A}^{(\text {weak })}(2 t)=\left\{\left(\begin{array}{lll}
0 & 1 & 1 \\
1 & 0 & 0 \\
0 & 0 & 0
\end{array}\right),\left(\begin{array}{lll}
0 & 1 & 1 \\
0 & 0 & 0 \\
1 & 0 & 0
\end{array}\right)\right\} .
$$

As $\lambda_{\mathrm{AS}}^{\text {(weak) }}=2>\lambda_{\min }=0$, the AS-algorithm is ineffective. Luckily, under variant 'extreme' both, the AS-scaling-sequence and the AS-seat-sequence remain unchanged once step 12 is reached,

$$
\begin{aligned}
& V^{(\operatorname{extr})}(11) \neq V^{(\operatorname{extr})}(12)=V^{(\operatorname{extr})}(13)=\cdots=\left(\begin{array}{ccc}
0.1 & 0.5 & 0.5 \\
0.5 & 0.5 & 0.5 \\
0.5 & 0.5 & 0.5
\end{array}\right) \\
& \mathcal{A}^{(\text {extr) }}(11) \neq \mathcal{A}^{(\operatorname{extr})}(12)=\cdots=\left\{\left(\begin{array}{lll}
0 & 0 & 1 \\
0 & 1 & 0 \\
1 & 0 & 0
\end{array}\right),\left(\begin{array}{cccc}
0 & 0 & 1 \\
1 & 0 & 0 \\
0 & 1 & 0
\end{array}\right),\left(\begin{array}{ccc}
0 & 1 & 0 \\
0 & 0 & 1 \\
1 & 0 & 0
\end{array}\right),\left(\begin{array}{ccc}
0 & 1 & 0 \\
1 & 0 & 0 \\
0 & 0 & 1
\end{array}\right)\right\} .
\end{aligned}
$$

Moreover the AS-limit-error coincides with the $L_{1}$-minimal-error, $\lambda_{\mathrm{AS}}^{(\text {extr) }}=0=\lambda_{\min }$. Hence, the AS-algorithm is effective. Above all it determines the set of biproportional apportionments, $\mathcal{A}(12)=\mathcal{B}(V, r, s)$, and a feasible set of divisors $\left(x^{(\mathrm{extr})}(12), y^{(\mathrm{extr})}(33)\right)$, where $x^{(\mathrm{extr})}(12)=$ $(1,0.4,0.4)$ and $y^{\text {(extr) }}(12)=(2.5,1,1)$. 
Our second example studies a problem that comes with a positive $L_{1}$-minimal-error. It turns out that the accumulation-points of AS-seat-sequence vary under different variants.

Example 2 (Accumulation-points). Let us take a look at the following biproportional problem:

$$
\begin{array}{cccccc} 
& & 1 & 3 & 5 & 11 \\
\cline { 2 - 6 }(V, r, s,\langle\cdot\rangle): & 2 & 1.1 & 1.3 & 0 & 0 \\
& 4 & 2.2 & 2.4 & 0 & 0 \\
& 6 & 1 & 1 & 2.6 & 2.8 \\
& 8 & 1 & 1 & 3.7 & 3.9
\end{array}
$$

For $I=\{1,2\}$ we have $r_{I}=6>4=s_{J_{V}(I)}$. Thus the flow-criterion (6) is violated and the $L_{1^{-}}$ minimal-error is positive, $\lambda_{\min }=4$. Under variant 'random' the AS-limit-error coincides with the $L_{1}$-minimal-error, $\lambda_{\mathrm{AS}}^{(\mathrm{rand})}=\lambda_{\min }=4$. The AS-scaling sequence and the AS-seat-sequence have seven accumulation-points each,

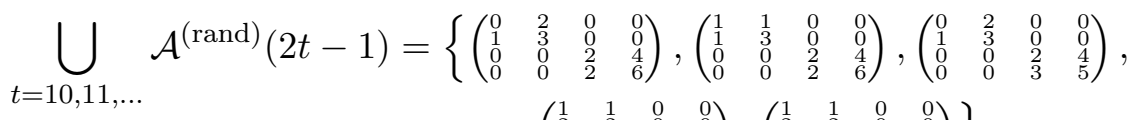

$$
\begin{aligned}
& \left.\left(\begin{array}{llll}
1 & 1 & 0 & 0 \\
2 & 2 & 0 & 0 \\
0 & 0 & 2 & 4 \\
0 & 0 & 2 & 6
\end{array}\right),\left(\begin{array}{llll}
1 & 1 & 0 & 0 \\
2 & 2 & 0 & 0 \\
0 & 0 & 2 & 4 \\
0 & 0 & 3 & 5
\end{array}\right)\right\}, \\
& \bigcup_{t=10,11, \ldots} \mathcal{A}^{(\text {rand })}(2 t)=\left\{\left(\begin{array}{cccc}
0 & 1 & 0 & 0 \\
0 & 2 & 0 & 0 \\
0 & 0 & 2 & 0 \\
0 & 0 & 3 & 6
\end{array}\right),\left(\begin{array}{cccc}
0 & 1 & 0 & 0 \\
1 & 2 & 0 & 0 \\
0 & 0 & 2 & 0 \\
0 & 0 & 3 & \frac{4}{7}
\end{array}\right)\right\} \text {. }
\end{aligned}
$$

Under variant 'midpoint' the AS-scaling-sequence and the AS-seat-sequence generate merely two accumulation-points each: those listed first respectively. As above $\lambda_{\mathrm{AS}}^{(\mathrm{mdpt})}=\lambda_{\min }=4$ holds and the AS-algorithm is effective.

Our third example points out that convergence of the AS-scaling-sequence does not necessarily imply convergence of the AS-seat-sequence.

Example 3 (Divergence \& convergence). Let us consider to following problem:

$$
\begin{array}{cccccccccc} 
& & 1 & 1 & 1 & 1 & 1 & 1 & 1 & 1 \\
\cline { 2 - 9 }(V, r, s,\langle\cdot\rangle): & 3 & 0.5 & 0.5 & 0.5 & 0.5 & 0 & 0 & 0 & 0 \\
& 3 & 0.5 & 0.5 & 0.5 & 0.5 & 0 & 0 & 0 & 0 \\
& 1 & 0.1 & 0.2 & 0.3 & 0.4 & 0.5 & 0.5 & 0.5 & 0.5 \\
& 1 & 0.4 & 0.3 & 0.2 & 0.1 & 0.5 & 0.5 & 0.5 & 0.5
\end{array}
$$

For $I=\{1,2\}$ we get $r_{I}=6>4=s_{J_{V}(I)}$. Again the flow-criterion is violated and the $L_{1^{-}}$ minimal-error is positive, $\lambda_{\min }=4$. In the first step all district-divisors-independent of the AS-algorithm's variant-are equal to one, $\rho_{1}(1)=\cdots=\rho_{4}(1)=1$. That is, for every districtadjustment at least two weights coincide with a signpost, of which one has to be rounded upwards and one has to be rounded downwards. This yields $V=V(1), f(1)=4$ and

$$
\left.\mathcal{A}(1)=\left\{\begin{array}{cccccccc}
1 & 1 & 1 & 0 & 0 & 0 & 0 & 0 \\
0 & 1 & 1 & 1 & 0 & 0 & 0 & 0 \\
0 & 0 & 0 & 0 & 1 & 0 & 0 & 0 \\
0 & 0 & 0 & 0 & 0 & 1 & 0 & 0
\end{array}\right), \begin{array}{c}
\text { +Ties } \\
\text { in blocks } \\
\{1,2\} \times\{1, \ldots, 4\} \\
\text { and } \\
\{3,4\} \times\{5,6,7,8\}
\end{array}\right\} .
$$

Analogously, in step two all incremental party-divisors are equal to one, $\sigma_{1}(2)=\cdots=\sigma_{8}(2)=$ 1 , yielding $V=V(1)=V(2), f(1)=f(2)=4$ and

$$
\mathcal{A}(2)=\left\{\left(\begin{array}{llllllll}
1 & 1 & 1 & 0 & 0 & 0 & 0 & 0 \\
0 & 0 & 0 & 1 & 0 & 0 & 0 & 0 \\
0 & 0 & 0 & 0 & 1 & 1 & 1 & 0 \\
0 & 0 & 0 & 0 & 0 & 0 & 0 & 1
\end{array}\right), \begin{array}{c}
\text { +Ties } \\
\text { in blecks } \\
\{1,2\} \times\{1, \ldots, 4\} \\
\text { and } \\
\{3,4\} \times\{5,6,7,8\}
\end{array}\right\} .
$$

Thus the AS-scaling-sequence $V(t)$ is constant and the AS-seat-sequence $\mathcal{A}(t)$ has two accumulation-points,

$$
V(t)=V \quad \forall t=1,2, \ldots \quad \text { and } \quad \mathcal{A}(t)=\left\{\begin{array}{llll}
\mathcal{A}(1) & \text { if } & t & \text { odd } \\
\mathcal{A}(2) & \text { if } & t & \text { even }
\end{array}\right.
$$

For the AS-limit-error we get $\lambda_{\mathrm{AS}}=4=\lambda_{\min }$, so that the AS-algorithm is effective. 
For the fourth example we modify the above example 3 such that the $L_{1}$-minimal-error vanishes. All the same, the AS-scaling-sequence $V(t)$ remains constant. However, this time the AS-limit-error is strictly larger than the $L_{1}$-minimal-error.

Example 4 (Ineffectivity I). Let us consider:

$$
\begin{array}{cccccccccc} 
& & 1 & 1 & 1 & 1 & 1 & 1 & 1 & 1 \\
\cline { 2 - 9 }(V, r, s,\langle\cdot\rangle): & 3 & 0.5 & 0.5 & 0.5 & 0.5 & 0.4 & 0.3 & 0.2 & 0.1 \\
& 3 & 0.5 & 0.5 & 0.5 & 0.5 & 0.1 & 0.2 & 0.3 & 0.4 \\
& 1 & 0.1 & 0.2 & 0.3 & 0.4 & 0.5 & 0.5 & 0.5 & 0.5 \\
& 1 & 0.4 & 0.3 & 0.2 & 0.1 & 0.5 & 0.5 & 0.5 & 0.5
\end{array}
$$

With all weights being positive the $L_{1}$-minimal-error vanishes, $\lambda_{\min }=0$. Analogously to example 3 the AS-scaling-sequence is constant and the AS-seat-sequence has two accumulationpoints. This yields $\lambda_{\mathrm{AS}}=4>0=\lambda_{\min }$ such that the AS-algorithm is ineffective.

Our last example illustrates that the AS-algorithm may also be ineffective if the $L_{1}$-minimalerror is positive.

Example 5 (Ineffectivity II). Let us consider:

\begin{tabular}{cc|cccccccc} 
& & 1 & 1 & 1 & 1 & 1 & 1 & 1 & 1 \\
\cline { 2 - 8 }$(V, r, s,\langle\cdot\rangle):$ & 3 & 0.5 & 0.5 & 0.5 & 0.5 & 0.1 & 0 & 0 & 0 \\
& 3 & 0.5 & 0.5 & 0.5 & 0.5 & 0.2 & 0 & 0 & 0 \\
& 1 & 0.1 & 0.2 & 0.3 & 0.4 & 0.5 & 0.5 & 0.5 & 0.5 \\
& 1 & 0.4 & 0.3 & 0.2 & 0.1 & 0.5 & 0.5 & 0.5 & 0.5
\end{tabular}

With $I=\{1,2\}$ we have $r_{I}=6>5=s_{J_{V}(I)}$ such that the $L_{1}$-minimal-error is positive, $\lambda_{\min }=2$. Analogously to examples 3 and 4 the AS-scaling-sequence is constant and the AS-seat-sequence has two accumulation-points. This yields $\lambda_{\mathrm{AS}}=4>2=\lambda_{\min }$ and the AS-algorithm is once more ineffective.

\section{5. $L_{1}$-minimal-error and AS-limit-error}

The upcoming proposition shows that the $L_{1}$-error of the AS-seat-sequence is monotonically decreasing and bounded from below by the $L_{1}$-minimal-error.

Definition 3 ( $L_{1}$-minimal-error). The $L_{1}$-minimal-error is defined by

$$
\lambda_{\min }:= \begin{cases}\max _{I \subseteq\{1, \ldots, k\}} 2\left(r_{I}-s_{J_{V}(I)}\right) & \text { if } \llbracket \cdot \rrbracket \text { pervious } \\ \max _{I \subseteq\{1, \ldots, k\}} 2\left(r_{I}+e_{I^{c} J_{V}(I)}-s_{J_{V}(I)}\right) & \text { if } \llbracket \cdot \rrbracket \text { impervious. }\end{cases}
$$

Note that the $L_{1}$-minimal-error vanishes if and only if the flow-criterion (6) is fulfilled. The upcoming proposition corrects Maier (2008). We show that the $L_{1}$-error of the AS-seat-sequence is monotonically decreasing.

Proposition 5 (Monotonicity). For the $L_{1}$-error of the $A S$-seat-sequence we have

$$
2 h \geq f(1) \geq f(t) \geq f(t+1) \geq \lambda_{\min } \quad \forall t=2,3, \ldots
$$

Proof. Without loss of generality let $A^{\prime} \in \mathcal{A}(2 t)$ be any apportionment with matching partyseats. The upper bound follows from

$$
f\left(A^{\prime}\right)=\sum_{i \leq k}\left|a_{i+}^{\prime}-r_{i}\right| \leq \sum_{i \leq k} a_{i+}^{\prime}+r_{+}=s_{+}+r_{+}=2 h
$$

To prove the lower bound let $I \subseteq\{1, \ldots, k\}$ be an arbitrary subset. We get

$$
f\left(A^{\prime}\right)=\sum_{i \in I}\left|r_{i}-a_{i+}^{\prime}\right|+\sum_{i \in I^{c}}\left|a_{i+}^{\prime}-r_{i}\right| \geq \underbrace{\sum_{i \in I}\left(r_{i}-a_{i+}^{\prime}\right)}_{(1)}+\underbrace{\sum_{i \in I^{c}}\left(a_{i+}^{\prime}-r_{i}\right)}_{(2)} .
$$


Rearranging expressions (1) and (2) results in

$$
\begin{aligned}
(1) & =r_{I}-a_{I J_{V}(I)}^{\prime}=r_{I}-\left(s_{J_{V}(I)}-a_{I^{c} J_{V}(I)}^{\prime}\right)=r_{I}-s_{J_{V}(I)}+a_{I^{c} J_{V}(I)}^{\prime} \\
(2) & =a_{I^{c} J_{V}(I)}^{\prime}+a_{I^{c} J_{V}(I)^{c}}^{\prime}-r_{I^{c}}=a_{I^{c} J_{V}(I)}^{\prime}+s_{J_{V}(I)^{c}}-\left(r_{+}-r_{I}\right) \\
& =a_{I^{c} J_{V}(I)}^{\prime}+\left(s_{+}-s_{J_{V}(I)}\right)-\left(r_{+}-r_{I}\right)=r_{I}-s_{J_{V}(I)}+a_{I^{c} J_{V}(I)}^{\prime} .
\end{aligned}
$$

For pervious rounding rules the lower bound follows as $f\left(A^{\prime}\right) \geq(1)+(2)=2\left(r_{I}-s_{J_{V}(I)}+\right.$ $\left.a_{I^{c} J_{V}(I)}^{\prime}\right) \geq 2\left(r_{I}-s_{J_{V}(I)}\right)$. For impervious rounding rules the assertion follows analogously from $a_{I^{c} J_{V}(I)}^{\prime} \geq e_{I^{c} J_{V}(I)}$

To prove monotonicity we study the passage from $\mathcal{A}(2 t-1)$ to $\mathcal{A}(2 t)$. If $f(2 t-1)=f(2 t)=$ $\lambda_{\text {min }}$ the situation is obvious. Otherwise let $A=\left(a_{i j}\right) \in \mathcal{A}(2 t-1)$ be any apportionment with matching district-contingents and let $f_{j}:=\left|a_{+j}-s_{j}\right|$ describe the $L_{1}$-error for party $j$. We show that for any apportionment $A^{\prime}=\left(a_{i j}^{\prime}\right) \in \mathcal{A}(2 t)$ and any fixed party $j$ the differences $a_{i j}-a_{i j}^{\prime}, i \leq k$, have the same sign. We first construct an apportionment $\bar{A}$ :

(1) Case $j \in J^{-}(2 t-1)$ : If $\sigma_{j}(2 t)<1$ the rounding rule's monotonicity implies $a_{i j} \leq \bar{a}_{i j}$ for all $\bar{a}_{i j} \in \llbracket v_{i j}(2 t) \rrbracket$ and all districts $i \leq k$.

If $\sigma_{j}(2 t)=1$ then $v_{i j}(2 t-1)=v_{i j}(2 t)$ for all $i \leq k$. Moreover there exist districts $i_{1}, \ldots, i_{f_{j}}$ such that the scaled votes are rounded downwards in step $2 t-1$ and rounded upwards in step $2 t$. Hence there exists an apportionment $\left(\bar{a}_{1 j}, \ldots, \bar{a}_{k j}\right) \in\left(\llbracket v_{1 j}(2 t) \rrbracket, \ldots\right.$, $\left.\llbracket v_{k j}(2 t) \rrbracket\right)$ with

$$
\bar{a}_{i j}:= \begin{cases}a_{i j}+1 & \text { if } i \in\left\{i_{1}, \ldots, i_{f_{j}}\right\} \\ a_{i j} & \text { otherwise. }\end{cases}
$$

Hence, the vector $\left(\bar{a}_{1 j}, \ldots, \bar{a}_{k j}\right)$ complies with the party-seats and $a_{i j} \leq \bar{a}_{i j}$ follows for all districts $i \leq k$.

(2) Case $j \in J^{+}(2 t-1)$ : The existence of some $\left(\bar{a}_{1 j}, \ldots, \bar{a}_{k j}\right) \in\left(\llbracket v_{1 j}(2 t) \rrbracket, \ldots, \llbracket v_{k j}(2 t) \rrbracket\right)$ with $\bar{a}_{+j}=s_{j}$ and $a_{i j} \geq \bar{a}_{i j}$ for all $i \leq k$ follows analogously to case (1).

(3) Case $j \in J^{=}(2 t-1)$ : With $\sigma_{j}(2 t)=1$ define $\left(\bar{a}_{1 j}, \ldots, \bar{a}_{k j}\right):=\left(a_{1 j}, \ldots, a_{k j}\right)$.

Hence $\bar{A}=\left(\bar{a}_{1 j}, \ldots, \bar{a}_{k j}\right)_{j \leq \ell}$ fulfills the party-seats and for any party $j$ the differences $a_{i j}-$ $\bar{a}_{i j}, i \leq k$, have the same sign. Therewith we get

$$
f(2 t-1)=f(A)=\sum_{j \leq \ell}\left|a_{+j}-s_{j}\right|=\sum_{j \leq \ell}\left|\sum_{i \leq k}\left(a_{i j}-\bar{a}_{i j}\right)\right|=\sum_{j \leq \ell} \sum_{i \leq k}\left|a_{i j}-\bar{a}_{i j}\right| .
$$

Finally the triangle inequality implies

$$
f(2 t-1)=\sum_{i \leq k} \sum_{j \leq \ell}\left|\bar{a}_{i j}-a_{i j}\right| \geq \sum_{i \leq k}\left|\sum_{j \leq \ell}\left(\bar{a}_{i j}-a_{i j}\right)\right|=\sum_{i \leq k}\left|\bar{a}_{i+}-r_{i}\right|=f(\bar{A}) \geq f(2 t) .
$$

The last inequality is due to the algorithm's definition, according to that only apportionments are considered that minimize the $L_{1}$-error. The passage from $\mathcal{A}(2 t)$ to $\mathcal{A}(2 t+1)$ follows analogously.

The $L_{1}$-error takes integer values only, it is bounded from below by the $L_{1}$-minimal-error and it is monotonically decreasing. For this reason there exists a minimal step such that the $L_{1}$-error remains unchanged thereafter. We call $t_{\mathrm{AS}}:=\min \{t \mid f(t)=f(t+1)=\cdots\}$ the AS-boundary-step. The $L_{1}$-error at the AS-boundary-step is the AS-limit-error.

Definition 4 (AS-limit-error). The AS-limit-error is defined by $\lambda_{\mathrm{AS}}:=f\left(t_{\mathrm{AS}}\right)$. 
The upcoming definition distinguishes between biproportional problems that come with an AS-limit-error that coincides with the $L_{1}$-minimal-error, and those whose AS-limit-error is strictly larger.

Definition 5 (Effectivity). The AS-algorithm is called effective if the AS-boundery-error coincides with the $L_{1}$-minimal-error, $\lambda_{\mathrm{AS}}=\lambda_{\min }$. Otherwise the AS-algorithm is called ineffective.

For the eight biproportional problems that arose in Switzerland between the years 2006 and 2012 the AS-algorithm is effective with all $L_{1}$-minimal-errors equal to zero. Simulations forebode that the AS-algorithm is always effective as long as the set of biproportional apportionments comes with at least a few ties (Balinski \& Pukelsheim, 2006; Gaffke \& Pukelsheim, 2008b). The following theorem substantiates this conjecture.

Theorem 1 (Effectivity). Let $(V, r, s, \llbracket \cdot \rrbracket)$ be a biproportional problem.

(1) Let the $L_{1}$-minimal-error vanish, $\lambda_{\min }=0$.

(i) If $k \cdot \ell<24$ holds for the number of districts and the number parties, then the $A S$-algorithm is effective.

(ii) If the set of biproportional apportionments comes with less than four ties, then the AS-algorithm is effective.

(2) Let the $L_{1}$-minimal-error be positive, $\lambda_{\min }>0$.

(i) If $k \cdot \ell<32$ holds for the number of districts and the number parties, then the AS-algorithm is effective.

(ii) If the sets $\mathcal{B}\left(V, r, s^{\prime}\right)$ and $\mathcal{B}\left(V, r^{\prime}, s\right)$ with $\sum_{i \leq k}\left|r_{i}^{\prime}-r_{i}\right|=\sum_{j \leq \ell}\left|s_{j}^{\prime}-s_{j}\right|=\lambda_{\min }$ each come with less than four ties, then the AS-algorithm is effective.

Proof. $\rightarrow$ (1i) and (2i): The proofs of part (1i) and part (2i) follow from the upper bound of the AS-limit-error, see section 8.

$\rightarrow$ (1ii) and (2ii): The proofs of part (1ii) and part (2ii) follow from the analysis of the ASTT-combination which may be applied in case the AS-algorithm is ineffective, see section 8.

\section{AS-seat-sequence}

If the AS-limit-error vanishes, $\lambda_{\mathrm{AS}}=0$, then the AS-algorithm determines the set of biproportional apportionments and the AS-seat-sequence converges, $\mathcal{A}\left(t_{\mathrm{AS}}\right)=\mathcal{A}\left(t_{\mathrm{AS}}+1\right)=\cdots=$ $\mathcal{B}(V, r, s)$. If the AS-limit-error is positive, $\lambda_{\mathrm{AS}}>0$, then the AS-algorithm generates apportionments that alternately fulfill district-contingents and party-seats. Hence there exist at least two accumulation-points. The upcoming theorem 2 subsumes a recapitulatory result on the convergence of the AS-seat-sequence. We distinguish between three cases. I: In case the ASalgorithm is effective and the $L_{1}$-minimal-error vanishes, the AS-seat-sequence converges. II: In case the AS-algorithm is effective and the $L_{1}$-minimal-error is positive, the AS-seat-sequence has at least two accumulation-points. III: In case the AS-algorithm is ineffective, the ASseat-sequence has-independent from the $L_{1}$-minimal-error - exactly two accumulation-points, $\mathcal{A}^{r}:=\lim _{\mathrm{t} \rightarrow \infty} \mathcal{A}(2 t-1)$ and $\mathcal{A}^{s}:=\lim _{\mathrm{t} \rightarrow \infty} \mathcal{A}(2 t)$.

Theorem 2 (AS-seat-sequence).

(1) Let the AS-algorithm be effective, $\lambda_{A S}=\lambda_{\min }$.

(i) If the $L_{1}$-minimal-error vanishes, $\lambda_{\min }=0$, then the $A S$-seat-sequence converges, $\mathcal{A}\left(t_{A S}\right)=\mathcal{A}\left(t_{A S}+1\right)=\cdots=\mathcal{B}(V, r, s)$.

(ii) If the $L_{1}$-minimal-error is positive, $\lambda_{\min }>0$, then the $A S$-seat-sequence has at least two accumulation-points. 
(2) Let the AS-algorithm be ineffective, $\lambda_{A S}>\lambda_{\min }$. The AS-seat-sequence has-independent from the $L_{1}$-minimal-error-two accumulation-points, $\mathcal{A}^{r}:=\lim _{\mathrm{t} \rightarrow \infty} \mathcal{A}(2 t-1)$ and $\mathcal{A}^{s}:=$ $\lim _{\mathrm{t} \rightarrow \infty} \mathcal{A}(2 t)$.

Proof. $\rightarrow$ (1i): The statement follows directly from the definition.

$\rightarrow$ (1ii): As the AS-limit-error is positive and district-contingents and party-seats are adjusted alternately, there exist at least two accumulation-points.

$\rightarrow$ (2): Theorem 3 hereafter shows that the AS-scaling-sequence $V(t)$ converges in case the AS-algorithm is ineffective. As the AS-limit-error is positive, $\lambda_{\mathrm{AS}}>0$, the existence of exactly two accumulation-points is established.

\section{AS-scaling-sequence}

If the AS-limit-error vanishes, $\lambda_{\mathrm{AS}}=0$, all incremental divisors are equal to one as soon as the AS-boundary-step $t_{\mathrm{AS}}$ is reached. Hence, not only the AS-seat-sequence $\mathcal{A}(t)$ but also the AS-scaling-sequence $V(t)$ converges. In general we have

Theorem 3 (AS-scaling-sequence). (1) Let the AS-algorithm be effective, $\lambda_{A S}=\lambda_{\min }$.

(i) If the $L_{1}$-minimal-error vanishes, $\lambda_{\min }=0$, the AS-scaling-sequence converges.

(ii) If $\lambda_{\min }>0$, then the AS-scaling-sequence either converges or diverges.

(2) Let the AS-algorithm be ineffective, $\lambda_{A S}>\lambda_{\min }$. The AS-scaling-sequence converges,

$$
t_{K}:=\min \{t \mid V(t)=V(t+1)=\cdots\}<\infty .
$$

We call $t_{K}$ the constant-step and $V\left(t_{K}\right)$ the boundary-matrix.

Proof. $\rightarrow$ (1i): The statement follows from the AS-algorithm's definition.

$\rightarrow$ (1ii): For a positive $L_{1}$-minimal-error examples 2 and 3 show that the AS-scaling-sequence either converges or diverges.

$\rightarrow$ (2): Let us first show that the set of under-represented districts and the set of overrepresented districts are disjoint for all $t, t^{\prime}>\left\lceil t_{\mathrm{AS}} / 2\right\rceil$. The same holds for the set of underrepresented parties and the set of over-represented parties,

$$
I^{-}(2 t) \cap I^{+}\left(2 t^{\prime}\right)=\emptyset \quad \text { and } \quad J^{-}(2 t-1) \cap J^{+}\left(2 t^{\prime}-1\right)=\emptyset .
$$

As $\lambda_{\mathrm{AS}}>0$ the sets $I^{-}(2 t), I^{+}(2 t), J^{-}(2 t-1)$, and $J^{+}(2 t-1)$ are not empty for all $t \geq\left\lceil t_{\mathrm{AS}} / 2\right\rceil$. Thus there exists an up\&down-cycle $\left(i_{(q)}, j_{(q)}\right)$ defined through

$$
\begin{array}{clll}
\left(i_{1}, j_{1}\right) \in I^{-}(2 t) & \times J^{+}(2 t+1) & \left(i_{2}, j_{1}\right) \in I^{-}(2 t+2) & \times J^{+}(2 t+1) \\
\left(i_{2}, j_{2}\right) \in I^{-}(2 t+2) \times J^{+}(2 t+3) & \left(i_{3}, j_{2}\right) \in I^{-}(2 t+4) & \times J^{+}(2 t+3) \\
& \ldots & \left(i_{1}, j_{q}\right) \in I^{-}(2 t+q+1) \times J^{+}(2 t+q) .
\end{array}
$$

Analogously there exists an up\&down-cycle $\left(i_{\left(q^{\prime}\right)}, j_{\left(q^{\prime}\right)}\right)$ such that

$$
\begin{array}{clll}
\left(i_{1}, j_{1}\right) \in I^{-}(2 t)^{c} \times J^{+}(2 t+1)^{c} & \left(i_{2}, j_{1}\right) \in I^{-}(2 t+2)^{c} & \times J^{+}(2 t+1)^{c} \\
\left(i_{2}, j_{2}\right) \in I^{-}(2 t+2)^{c} \times J^{+}(2 t+3)^{c} & \left(i_{3}, j_{2}\right) \in I^{-}(2 t+4)^{c} & \times J^{+}(2 t+3)^{c} \\
& & \left(i_{1}, j_{q^{\prime}}\right) \in I^{-}\left(2 t+q^{\prime}+1\right)^{c} \times J^{+}\left(2 t+q^{\prime}\right)^{c} .
\end{array}
$$

Given the proof of proposition 5 we know that for any fixed party $j$ the differences $\left(a_{i j}-a_{i j}^{\prime}\right), i \leq$ $k$, all carry the same sign. With $f(A)=f\left(A^{\prime}\right)$ the triangular inequality (9) holds with equality,

$$
\sum_{i \leq k} \sum_{j \leq \ell}\left|a_{i j}^{\prime}-a_{i j}\right|=\sum_{i \leq k}\left|\sum_{j \leq \ell} a_{i j}^{\prime}-a_{i j}\right| .
$$


Thus also for any fixed district $i$ the differences $\left(a_{i j}-a_{i j}^{\prime}\right), j \leq \ell$, all carry the same sign. Hence, for all up\&down-cycles $\left(i_{(q)}, j_{(q)}\right)$ and $\left(i_{\left(q^{\prime}\right)}, j_{\left(q^{\prime}\right)}\right)$ the row-sets are mutually disjoint, $I^{-}(2 t+p) \cap I^{-}\left(2 t+p^{\prime}\right)^{c}=\emptyset$ for all $p, p^{\prime} \leq q$. The same holds for the column-sets, $J^{+}(2 t+p) \cap$ $J^{+}\left(2 t+p^{\prime}\right)^{c}=\emptyset$ for all $p, p^{\prime} \leq q$. This establishes (10).

Let us next define the non-trivial set of districts that are under-represented once the ASboundary-step is reached. Analogously we define the non-trivial set of parties that are overrepresented,

$$
I^{-}:=\bigcup_{t>\left\lceil t_{\mathrm{AS}} / 2\right\rceil} I^{-}(2 t) \quad \text { and } \quad J^{+}:=\bigcup_{t>\left\lceil t_{\mathrm{AS}} / 2\right\rceil} J^{+}(2 t-1) .
$$

With equations $(7),(8)$ and (10) we get

$$
\rho_{i}(2 t-1)\left\{\begin{array} { l l } 
{ \leq 1 } & { \text { if } i \in I ^ { - } , } \\
{ \geq 1 } & { \text { if } i \in ( I ^ { - } ) ^ { c } , }
\end{array} \sigma _ { j } ( 2 t ) \left\{\begin{array}{ll}
\leq 1 & \text { if } j \in\left(J^{+}\right)^{c} \\
\geq 1 & \text { if } j \in J^{+}
\end{array}\right.\right.
$$

To complete the proof suppose that the AS-scaling-sequence $V(t)$ diverges. With equation (11) the entries of the AS-scaling-sequence $V(t)$ are monotonically increasing in block $I^{-} \times\left(J^{+}\right)^{c}$, and monotonically decreasing in block $\left(I^{-}\right)^{c} \times J^{+}$. However, the entries are bounded from below and above. Hence, for any apportionment $A=\left(a_{i j}\right) \in \mathcal{A}(2 t-1), t>\left\lceil t_{\mathrm{AS}} / 2\right\rceil$, we get

$$
v_{i j}=a_{i j}=0 \quad \forall(i, j) \in I^{-} \times\left(J^{+}\right)^{c}, \quad a_{i j}=\left\{\begin{array}{ll}
0 & \text { if } \llbracket \cdot \rrbracket \text { pervious } \\
e_{i j} & \text { if } \llbracket \cdot \rrbracket \text { impervious }
\end{array} \quad \forall(i, j) \in\left(I^{-}\right)^{c} \times J^{+} .\right.
$$

As the $L_{1}$-error of $A$ coincides with the AS-limit-error we get

$$
\begin{aligned}
\lambda_{\mathrm{AS}}=f(A) & = \begin{cases}2\left(r_{I^{-}}-s_{J_{V}\left(I^{-}\right)}\right) & \text {if } \llbracket \cdot \rrbracket \text { pervious } \\
2\left(r_{I^{-}}+e_{\left(I^{-}\right)^{c} J_{V}\left(I^{-}\right)}-s_{J_{V}\left(I^{-}\right)}\right) & \text {if } \llbracket \cdot \rrbracket \text { impervious, }\end{cases} \\
& \leq\left\{\begin{array}{ll}
\max _{I \subseteq\{1, \ldots, k\}} 2\left(r_{I}-s_{J_{V}(I)}\right) & \text { if } \llbracket \cdot \rrbracket \text { pervious } \\
\max _{I \subseteq\{1, \ldots, k\}} 2\left(r_{I}+e_{I^{c} J_{V}(I)}-s_{J_{V}(I)}\right) & \text { if } \llbracket \cdot \rrbracket \text { impervious, }
\end{array}=\lambda_{\min } .\right.
\end{aligned}
$$

This is a contradiction to the AS-algorithm's ineffectiveness, i.e. to $\lambda_{\mathrm{AS}}>\lambda_{\min }$.

To be safeguarded against ineffectivity the following lemma transpires to be an essential tool.

$$
V(2 t-2):\left(\begin{array}{c|ccc} 
& J^{+} & \left(J^{+}\right)^{c} & \\
\cline { 1 - 3 } I^{-} & * & \uparrow & : \rho_{i}(2 t-1) \leq 1 \\
\left(I^{-}\right)^{c} & \downarrow & * & : \rho_{i}(2 t-1) \geq 1
\end{array}\right) \forall t>\left\lceil t_{\mathrm{AS}} / 2\right\rceil .
$$

Figure 1: $A S$-scaling-sequence in case $\lambda_{A S}>0$. For all steps $t \geq t_{\mathrm{AS}}$ entries labeled with ' $\uparrow$ ' are monotonically increasing, while entries labeled with ' $\downarrow$ ' are monotonically decreasing. Entries labeled with ' $*$ ' are alternately increasing and decreasing. As entries are bounded from below and from above, there either exists a step $t_{K}$ with $V\left(t_{K}\right)=V\left(t_{K}+1\right)=\cdots$, or entries labeled with ' $\downarrow$ ' converge to 0 (resp. $e_{i j}$ in case $\llbracket \cdot \rrbracket$ is impervious) and for entries labeled with ' $\uparrow$ ' we have $v_{i j}=0$. Thus accumulation-points of the AS-seat-sequence only differ in ' $*$ '-entries. 
Lemma 1. Let the AS-algorithm be ineffective.

(1) For any apportionment $A=\left(a_{i j}\right) \in \mathcal{A}^{r}$ we have

$$
\begin{array}{llll}
\text { (i) } \quad \forall i \in I^{-} & \exists j_{1}, j_{2} \in J^{+}: v_{i j_{1}}\left(t_{K}\right)=s\left(a_{i j_{1}}\right), & v_{i j_{2}}\left(t_{K}\right)=s\left(a_{i j_{2}}+1\right), \\
\text { (ii) } \quad \forall j \in J^{+} \quad \exists i \in I^{-}: & v_{i j}\left(t_{K}\right)=s\left(a_{i j}\right) .
\end{array}
$$

(2) For any apportionment $A^{\prime}=\left(a_{i j}^{\prime}\right) \in \mathcal{A}^{s}$ we have

$$
\begin{aligned}
& \text { (i) } \forall i \in I^{-} \quad \exists j \in J^{+}: \quad v_{i j}\left(t_{K}\right)=s\left(a_{i j}^{\prime}+1\right) \text {, } \\
& \text { (ii) } \forall j \in J^{+} \quad \exists i_{1}, i_{2} \in I^{-}: v_{i_{1} j}\left(t_{K}\right)=s\left(a_{i_{1} j}^{\prime}\right), \quad v_{i_{2} j}\left(t_{K}\right)=s\left(a_{i_{2} j}^{\prime}+1\right) \text {. }
\end{aligned}
$$

Analogous assertions hold for the sets of over-represented districts $I^{+}:=\bigcup_{t>\left\lceil t_{A S} / 2\right\rceil} I^{+}(2 t)$ and under-represented parties $J^{-}:=\bigcup_{t>\left\lceil t_{A S} / 2\right\rceil} J^{-}(2 t-1)$.

Proof. The proof exploits that all apportionments $A, \tilde{A} \in \mathcal{A}^{r}$ and $A^{\prime}, \bar{A} \in \mathcal{A}^{s}$ emerge from the boundary-matrix $V\left(t_{K}\right)$ and that they only differ on the blocks $I^{-} \times J^{+}$and $\left(I^{-}\right)^{c} \times\left(J^{+}\right)^{c}$,

$$
\begin{aligned}
a_{i j}, \tilde{a}_{i j}, a_{i j}^{\prime}, \bar{a}_{i j} \in \llbracket v_{i j}\left(t_{K}\right) \rrbracket & \forall i \leq k, \forall j \leq \ell, \\
a_{i j}=\tilde{a}_{i j}=a_{i j}^{\prime}=\bar{a}_{i j} & \forall(i, j) \in I^{-} \times\left(J^{+}\right)^{c} \cup\left(I^{-}\right)^{c} \times J^{+} .
\end{aligned}
$$

$\rightarrow$ (1i): Let $i \in I^{-}$be an under-represented district. Then there exists an apportionment $\bar{A}=\left(\bar{a}_{i j}\right) \in \mathcal{A}^{s}$ such that $\bar{a}_{i+}<r_{i}$. With equation (12) there exists a party $j_{1} \in J^{+}$such that $v_{i j_{1}}=s\left(a_{i j_{1}}\right)=s\left(\bar{a}_{i j_{1}}+1\right)$. Due to the constant AS-scaling-sequence there exists another party $j_{2}$ such that $v_{i j_{2}}=s\left(a_{i j_{2}}+1\right)$. As the $L_{1}$-error is minimal we have $j_{2} \in J^{+}$.

$\rightarrow$ (1ii): Let $j \in J^{+}$be an over-represented party. If $j \in J^{+}(A)$, then $a_{+j}>s_{j}$ holds and with equation (12) there exists a district $i \in I^{-}$such that $v_{i j}=s\left(a_{i j}\right)$. If $j \in J^{=}(A)$, then $s_{j}=a_{+j}$ holds and there exists an apportionment different from $A, A \neq \tilde{A}=\left(\tilde{a}_{i j}\right) \in \mathcal{A}^{r}$ such that $j \in J^{+}(\tilde{A})$ and $\tilde{a}_{+j}>a_{+j}=s_{j}$. With equation (12) there exists a district $i_{1} \in I^{-}$such that $v_{i_{1} j}=s\left(\tilde{a}_{i_{1} j}\right)=s\left(a_{i_{1} j}-1\right)$. Due to the constant AS-scaling-sequence and the minimal $L_{1}$-error there exists another district $i_{2} \in I^{-}$such that $v_{i_{2} j}=s\left(a_{i_{2} j}\right)$.

$\rightarrow$ (2): Analogously to (1).

\section{Ineffectiveness-error}

In section 5 we have seen that the AS-limit-error is bounded from below by the $L_{1}$-minimalerror, $\lambda_{\mathrm{AS}} \geq \lambda_{\min }$. In this section we give an upper bound for the AS-limit-error.

Definition 6 (Ineffectiveness-error). The ineffectiveness-error is defined by

$$
\Lambda_{\text {ineff }}^{\mathrm{k}, \ell}:= \begin{cases}0 & \text { if } \min \{k, \ell\} \leq 3, \\ 2(\lceil\ell / 2\rceil(\lfloor k / 2\rfloor-1))-\lfloor k / 2\rfloor & \text { if }\lceil\ell / 2\rceil(\lfloor k / 2\rfloor-1) \leq\lceil k / 2\rceil(\lfloor\ell / 2\rfloor-1), \\ 2(\lceil k / 2\rceil)(\lfloor\ell / 2\rfloor-1)-\lfloor\ell / 2\rfloor & \text { otherwise. }\end{cases}
$$

For all $3 \leq k \leq 16$ and all $4 \leq \ell \leq 16$ the ineffectiveness-error is displayed in table 3 .

Proposition 6 (Ineffectiveness-error). The AS-limit-error is bounded from above by the maximum of the ineffectiveness-error and $L_{1}$-minimal-error, $\lambda_{A S} \leq \max \left\{\Lambda_{\text {ineff }}^{\mathrm{k}, \ell}, \lambda_{\min }\right\}$.

Proof. In case the AS-algorithm is effective, $\lambda_{\mathrm{AS}}=\lambda_{\min }$, nothing is to be shown. Let the ASalgorithm be ineffective and let $A \in \mathcal{A}^{r}$ and $A^{\prime} \in \mathcal{A}^{s}$ be two apportionments. The AS-limit-error may be paraphrased as

$$
\lambda_{\mathrm{AS}}=2\left(a_{I^{-} J^{+}}-a_{I^{-} J^{+}}^{\prime}\right) .
$$


We maximize the right hand side of equation (13) under the condition that $A$ and $A^{\prime}$ both emerge from the constant boundary-matrix $V\left(t_{K}\right)$. For any district $i \in I^{-}$there must exist at least one party $j \in J^{+}$such that the boundary-weight $v_{i j}\left(t_{K}\right)$ coincides with a signpost and is rounded downwards. Consequently, there are at most $\# I^{-}\left(\# J^{+}-1\right)$ weights $v_{i j}\left(t_{K}\right)$ in block $I^{-} \times J^{+}$that coincide with a signpost and are rounded upwards. Here, \#I and \#J indicate, respectively, the number of elements of any subset $I \subseteq\{1, \ldots, k\}$ and $J \subseteq\{1, \ldots, \ell\}$. Analogously, for every party $j \in J^{+}$there must exist at least one district $i \in I^{-}$such that the boundary-weight $v_{i j}\left(t_{K}\right)$ coincides with a signpost and is rounded upwards. Thus in block $I^{-} \times J^{+}$at least $\# J^{+}$weights that coincide with a signpost are rounded upwards. This yields $\lambda_{\mathrm{AS}} \leq 2\left(\# I^{-}\left(\# J^{+}-1\right)-\# J^{+}\right)$. Analogously we get $\lambda_{\mathrm{AS}} \leq 2\left(\# J^{-}\left(\# I^{+}-1\right)-\# I^{+}\right)$. Maximization over $\# I^{-}, \# I^{+}$and $\# J^{-}, \# J^{+}$establishes the assertion.

With proposition 6 the proof of theorem 1(1i, 2i) follows immediately.

Proof of theorem 1. $\rightarrow(1 i)$ : Proposition 6 yields $\lambda_{\mathrm{AS}} \leq \max \left\{\Lambda_{\text {ineff }}^{\mathrm{k}, \ell}, \lambda_{\min }\right\}=\Lambda_{\text {ineff }}^{\mathrm{k}, \ell}$ for all biproportional problems with vanishing $L_{1}$-minimal-error. For $k \cdot \ell<24$ we have $\Lambda_{\text {ineff }}^{\mathrm{k}, \ell}=0$. Hence, the AS-algorithm is effective.

$\rightarrow$ (2i): For $k \cdot \ell<32$ we have $\Lambda_{\text {ineff }}^{\mathrm{k}, \ell} \leq 2$. As the $L_{1}$-minimar-error is positive we get $\lambda_{\mathrm{AS}} \leq \max \left\{\Lambda_{\text {ineff }}^{\mathrm{k}, \ell}, \lambda_{\min }\right\}=\lambda_{\min }$. With $\lambda_{\mathrm{AS}} \geq \lambda_{\min }$ the assertion follows.

\begin{tabular}{r|rrrrrrrrrrrrr} 
& $\ell=4$ & 5 & 6 & 7 & 8 & 9 & 10 & 11 & 12 & 13 & 14 & 15 & 16 \\
\hline$k=3$ & 0 & 0 & 0 & 0 & 0 & 0 & 0 & 0 & 0 & 0 & 0 & 0 & 0 \\
4 & 0 & 0 & 2 & 2 & 4 & 6 & 6 & 8 & 8 & 10 & 10 & 12 & 12 \\
5 & & 2 & 2 & 4 & 4 & 6 & 6 & 8 & 8 & 10 & 10 & 12 & 12 \\
6 & & & 6 & 6 & 10 & 10 & 14 & 14 & 18 & 22 & 22 & 26 & 26 \\
7 & & & & 10 & 10 & 14 & 14 & 18 & 18 & 22 & 22 & 26 & 26 \\
8 & & & & & 16 & 16 & 22 & 22 & 28 & 28 & 34 & 34 & 40 \\
9 & & & & & & 22 & 22 & 28 & 28 & 34 & 34 & 40 & 40 \\
10 & & & & & & & 30 & 30 & 38 & 38 & 46 & 46 & 54 \\
11 & & & & & & & & 38 & 38 & 46 & 46 & 54 & 54 \\
12 & & & & & & & & & 48 & 48 & 58 & 58 & 68 \\
13 & & & & & & & & & & 58 & 58 & 68 & 68 \\
14 & & & & & & & & & & & 70 & 70 & 82 \\
15 & & & & & & & & & & & & 82 & 82 \\
16 & & & & & & & & & & & & &
\end{tabular}

Table 3: Ineffectiveness-error $\Lambda_{\text {ineff }}^{\mathrm{k}, \ell}$. The AS-limit-error is bounded from above. Proposition 6 establishes $\lambda_{\mathrm{AS}} \leq \max \left\{\Lambda_{\text {ineff }}^{\mathrm{k}, \ell}, \lambda_{\min }\right\}$.

\section{AS-TT-combination}

In case of effectivity the AS-algorithm determines a biproportional apportionment if and only if the flow-criterion is accomplished. Otherwise the AS-TT-combination, introduced hereafter, generates a biproportional apportionment if and only if the flow-criterion is true. Moreover, the analysis of the AS-TT-combination is the crux for the owing proofs of proposition 4 and theorem 1.

In linear programming the AS-algorithm is a cyclic coordinate method. That is, in every iteration the generated apportionments either comply with the district-contingents or with the party-seats. For cyclic coordinate methods criterion for convergence are well known. Bazaraa, Sherali \& Shetty (2006, page 285) (see also Gaffke \& Pukelsheim (2008b) in connection with the AS-algorithm) show that a cyclic coordinate method converges if every step is unambiguous. To circumvent ineffectivity Bazaraa, Sherali \& Shetty (2006, page 287) advocate a pattern-searchstep. We extracts such a step from Balinski \& Demange's (1989b) Tie\&Transfer-algorithm.

Let us first take a look at the hybrid AS-TT-algorithm introduced by Maier (2008). Simulations by Maier et al. (2010) suggest that the set of biproportional apportionments can be determined as fast as possible if the procedure is initialized by the AS-algorithm and switches to the Tie\&Transfer-algorithm as soon as the $L_{1}$-error remains unchanged for two steps. 
Firstly because run times do not matter for practical purposes, and secondly because the Tie\&Transfer-algorithm is less intuitive than the AS-algorithm, we suggest to switch only in case of the AS-algorithm's ineffectivity. We call this option the AS-TT-combination. Similar to the AS-algorithm, the AS-TT-combination generates sequences of apportionments, scaled weights, incremental and cumulative divisors, under-represented districts and over-represented parties. We label those objects with an index $T$, e.g. $\mathcal{A}_{T}(n)$ denoting the AS-TT-seat-sequence.

Let the AS-algorithm be ineffective, let $V\left(t_{K}\right)$ be the boundary-matrix, and let $\mathcal{A}^{r}$ and $\mathcal{A}^{s}$ denote the two accumulation-points of the AS-seat-sequence. The AS-TT-combination is initialized by $\mathcal{A}_{T}^{r}(0):=\mathcal{A}^{r}, \mathcal{A}_{T}^{s}(0):=\mathcal{A}^{s}, \mathcal{A}_{T}(0):=\mathcal{A}^{r} \cup \mathcal{A}^{s}, V_{T}(0):=V\left(t_{K}\right), I_{T}^{-}(0):=I^{-}$ and $J_{T}^{+}(0):=J^{+}$. In the first step the weight-matrix $V_{T}(0)$ is scaled such that entries $(i, j) \in$ $I_{T}^{-}(0) \times J_{T}^{+}(0)^{c}$ are increased and entries $(i, j) \in I_{T}^{-}(0)^{c} \times J_{T}^{+}(0)$ are decreased, while the remaining entries are not modified and the two accumulation-points of the AS-seat-sequence may still be determined by rounding the scaled weights (see figure 2). Moreover, there shall exist an entry $(i, j) \in I_{T}^{-}(0) \times J_{T}^{+}(0)^{c}$ that after being scaled coincides with the signpost next in size, or an entry $(i, j) \in I_{T}^{-}(0)^{c} \times J_{T}^{+}(0)$ that after being scaled coincides with the next smaller signpost. To this end let $A \in \mathcal{A}(0)$ be any apportionment and define $\varepsilon(1):=\max \left(\varepsilon(1)^{+}, \varepsilon(1)^{-}\right)$ through

$$
\varepsilon(1)^{+}:=\max _{(i, j) \in I_{T}^{-}(0) \times J_{T}^{+}(0)^{c}} \frac{v_{i j}^{T}(0)}{s\left(a_{i j}+1\right)}, \quad \varepsilon(1)^{-}:=\max _{(i, j) \in I_{T}^{-}(0)^{c} \times J_{T}^{+}(0)} \frac{s\left(a_{i j}\right)}{v_{i j}^{T}(0)} .
$$

By theorem 2 all apportionments $A \in \mathcal{A}(0)$ coincide on $I^{-} \times\left(J^{+}\right)^{c}$ and $\left(I^{-}\right)^{c} \times J^{+}$. Hence, $\varepsilon(1)$ does not depend on any particular $A \in \mathcal{A}(0)$. We define the vote-matrix $V_{T}(1)=\left(v_{i j}^{T}(1)\right)$ by

$$
v_{i j}^{T}(1):= \begin{cases}v_{i j}^{T}(0) / \varepsilon(1) & \text { if }(i, j) \in I_{T}^{-}(0) \times J_{T}^{+}(0)^{c} \\ v_{i j}^{T}(0) \cdot \varepsilon(1) & \text { if }(i, j) \in I_{T}^{-}(0)^{c} \times J_{T}^{+}(0), \\ v_{i j}^{T}(0) & \text { otherwise. }\end{cases}
$$

Subsequently we identify those apportionments that are obtained from $V_{T}(1)$ by rounding, that minimize the $L_{1}$-error, and either comply with all district-contingents or all party-seats. That is, we define $\mathcal{A}_{T}(1):=\mathcal{A}_{T}^{r}(1) \cup \mathcal{A}_{T}^{s}(1)$, where

$$
\begin{aligned}
& \mathcal{A}_{T}^{r}(1):=\left\{A=\left(a_{i j}\right) \mid a_{i j} \in \llbracket v_{i j}^{T}(1) \rrbracket, \quad a_{i+}=r_{i}, \quad f(A)=\min _{B=\left(b_{i j}\right), b_{i j} \in \llbracket v_{i j}^{T}(1) \rrbracket} f(B)\right\}, \\
& \mathcal{A}_{T}^{s}(1):=\left\{A=\left(a_{i j}\right) \mid a_{i j} \in \llbracket v_{i j}^{T}(1) \rrbracket, \quad a_{+j}=s_{j}, \quad f(A)=\min _{B=\left(b_{i j}\right), b_{i j} \in \llbracket v_{i j}^{T}(1) \rrbracket}^{B=\left(b_{i j}\right), b_{i j} \in \llbracket v_{i j}^{T}(1) \rrbracket} f(B)\right\} .
\end{aligned}
$$

To conclude, we identify the sets of under-represented districts and over-represented parties,

$$
I_{T}^{-}(1):=\bigcup_{A \in \mathcal{A}_{T}^{s}(1)} I^{-}(A) \quad \text { and } \quad J_{T}^{+}(1):=\bigcup_{A \in \mathcal{A}_{T}^{r}(1)} J^{+}(A) .
$$

Note that all apportionments $A \in \mathcal{A}_{T}(1)$ come with the same $L_{1}$-error. Thus the $L_{1}$-error $f_{T}(1):=f(A)$ is well-defined.

Lemma 2. (1) For $\varepsilon(1)$ we get $\varepsilon(1)>0$.

(2) The $L_{1}$-error in step one is not larger than the AS-limit-error, $f_{T}(1) \leq \lambda_{A S}$. 


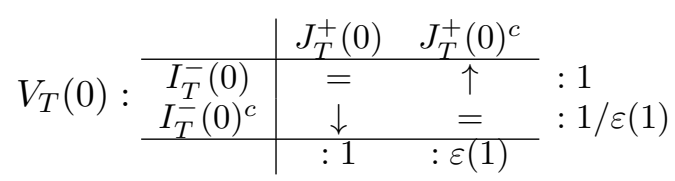

Figure 2: $A S$-TT-combination. $\varepsilon(1)$ is determined in order to increase the entries labeled with ' $\uparrow$ ', to decrease the entries labeled with ' $\downarrow$ ', and to leave entries labeled with '=' unchanged.

Proof. $\rightarrow$ (1): Let $A \in \mathcal{A}^{s} \subset \mathcal{A}(0)$ be any apportionment with adjusted party-seats. Suppose $\varepsilon(1)^{+}=\varepsilon(1)^{-}=0$. With $\varepsilon(1)^{-}=0$ we get $a_{\left(I^{-}\right)^{c} J^{+}}=0$ in case of a pervious rounding-rule and $a_{\left(I^{-}\right)^{c} J^{+}}=e_{\left(I^{-}\right)^{c} J^{+}}$in case of an impervious rounding-rule. This yields

$$
\lambda_{\mathrm{AS}}=2 \sum_{i \in I^{-}}\left(r_{i}-a_{i+}\right)=\left\{\begin{array}{ll}
2\left(r_{I^{-}}-s_{J_{V}\left(I^{-}\right)}\right) & \text {if } \llbracket \cdot \rrbracket \text { pervious, } \\
2\left(r_{I^{-}}-s_{J_{V}\left(I^{-}\right)}+e_{\left(I^{-}\right)^{c} J^{+}}\right) & \text {if } \llbracket \cdot \rrbracket \text { impervious, }
\end{array} \leq \lambda_{\min } .\right.
$$

This however contradicts to the ineffectivity of the AS-algorithm, i.e. $\lambda_{\mathrm{AS}}>\lambda_{\min }$.

$\rightarrow$ (2): By definition we have $s\left(a_{i j}\right) \leq v_{i j}^{T}(1) \leq s\left(a_{i j}+1\right)$ for all districts $i \leq k$, all parties $j \leq \ell$, and all apportionments $A \in \mathcal{A}_{T}(0)$. Thus all apportionments $A \in \mathcal{A}(0)$ may also be obtained from $V_{T}(1)$ by rounding and $f_{T}(1) \leq \lambda_{\mathrm{AS}}$ follows trivially.

The following proposition shows that the AS-scaling-sequence is also constant for the biproportional problem that emerges from the first AS-TT-step. In addition, it states that all generated apportionments come with at least four ties.

Algorithm 2 The $A S$-TT-combination determines the set of biproportional apportionments in case the AS algorithm is ineffective. AS-TT steps trace back to Balinski \& Demange (1989b).

Initialization: $\operatorname{Set} \mathcal{A}_{T}^{r}(0):=\mathcal{A}^{r}, \mathcal{A}_{T}^{s}(0):=\mathcal{A}^{s}, \mathcal{A}_{T}(0):=\mathcal{A}^{r} \cup \mathcal{A}^{r}, V_{T}(0):=V\left(t_{K}\right), I_{T}^{-}(0):=I^{-}$and $J_{T}^{+}(0):=J^{+}$.

AS-TT step $n=1,2, \ldots$ : Let $A \in \mathcal{A}_{T}(n-1)$ be chosen arbitrary. Determine the scaled vote-matrix $V_{T}(n)=\left(v_{i j}^{T}(n)\right)$ defined by

$$
v_{i j}^{T}(n):= \begin{cases}v_{i j}^{T}(n-1) / \varepsilon(n) & \text { if }(i, j) \in I_{T}^{-}(n-1) \times J_{T}^{+}(n-1)^{c} \\ v_{i j}^{T}(n-1) \cdot \varepsilon(n) & \text { if }(i, j) \in I_{T}^{-}(n-1)^{c} \times J_{T}^{+}(n-1) \\ v_{i j}^{T}(n-1) & \text { otherwise }\end{cases}
$$

for $\varepsilon(n):=\max \left(\varepsilon(n)^{+}, \varepsilon(n)^{-}\right)$, where

$$
\varepsilon(n)^{+}:=\max _{(i, j) \in I_{T}^{-}(n-1) \times J_{T}^{+}(n-1)^{c}} \frac{v_{i j}^{T}(n-1)}{s\left(a_{i j}+1\right)}, \quad \varepsilon(n)^{-}:=\max _{(i, j) \in I_{T}^{-}(n-1)^{c} \times J_{T}^{+}(n-1)} \frac{s\left(a_{i j}\right)}{v_{i j}^{T}(n-1)} .
$$

Identify $\mathcal{A}_{T}(n):=\mathcal{A}_{T}^{r}(n) \cup \mathcal{A}_{T}^{s}(n)$ where

$$
\begin{aligned}
& \mathcal{A}_{T}^{r}(n):=\left\{A=\left(a_{i j}\right) \mid a_{i j} \in \llbracket v_{i j}^{T}(n) \rrbracket, \quad a_{i+}=r_{i}, \quad f(A)=\min _{B=\left(b_{i j}\right), b_{i j} \in \llbracket v_{i j}^{T}(n) \rrbracket} f(B)\right\}, \\
& \mathcal{A}_{T}^{s}(n):=\left\{A=\left(a_{i j}\right) \mid a_{i j} \in \llbracket v_{i j}^{T}(n) \rrbracket, \quad a_{+j}=s_{j}, \quad f(A)=\min _{B=\left(b_{i j}\right), b_{i j} \in \llbracket v_{i j}^{T}(n) \rrbracket} f(B)\right\} .
\end{aligned}
$$

Define the set of under-represented districts and over-represented parties

$$
I_{T}^{-}(1):=\bigcup_{A \in \mathcal{A}_{T}^{s}(1)} I^{-}(A) \quad \text { and } \quad J_{T}^{+}(1):=\bigcup_{A \in \mathcal{A}_{T}^{r}(1)} J^{+}(A) .
$$

Set $f_{T}(n):=f(A)$ for any apportionment $A \in \mathcal{A}_{T}(n)$.

If $f_{T}(n)=\lambda_{\min }$ set $n_{\mathrm{AS}-\mathrm{TT}}:=n$ and break. If $f_{T}(n)>\lambda_{\min }$ run AS-TT-step for $n:=n+1$.

Output: AS-TT-seat-sequence $\mathcal{A}_{T}(n)$, AS-TT-scaling-sequence $V_{T}(n)$ and sequence of divisors $\varepsilon(n)$. 


\section{Proposition 7 (AS-TT-step).}

(1) The AS-scaling-sequence is constant for the biproportional problem $\left(V_{T}(1), r, s, \llbracket \cdot \rrbracket\right)$.

(2) For all apportionments $A \in \mathcal{A}_{T}(1)$ there exist two cycles such that both admit a seattransfer. Thus $\# \mathcal{A}_{T}(1) \geq 4$ holds.

Proof. $\rightarrow(1):$ Without loss of generality let $\varepsilon(1)=\varepsilon(1)^{+} \geq \varepsilon(1)^{-}$. Hence there exists a cell $\left(i_{1}, j_{1}\right) \in I_{T}^{-}(0) \times J_{T}^{+}(0)^{c}$ such that $v_{i_{1} j_{1}}^{T}(1)=v_{i_{1} j_{1}}^{T}(0) / \varepsilon(1)=v_{i_{1} j_{1}}^{T}(0) / \varepsilon(1)^{+}=s\left(a_{i_{1} j_{1}}+1\right)$ for all $A \in \mathcal{A}_{T}(0)$. By definition we get

$$
v_{i j}^{T}(1)=v_{i j}^{T}(0) \quad \forall(i, j) \in I_{T}^{-}(0) \times J_{T}^{+}(0) \cup I_{T}^{-}(0)^{c} \times J_{T}^{+}(0)^{c} .
$$

By Lemma 1 and equation (14) there exists a party $j_{2} \in J_{T}^{+}(0)$ and an apportionment $A=$ $\left(a_{i j}\right) \in \mathcal{A}_{T}^{r}(0)$, such that $j_{2} \in J^{+}(A)$ and $v_{i_{1} j_{2}}^{T}(1)=v_{i_{1} j_{2}}^{T}(0)=s\left(a_{i_{1} j_{2}}\right)$. For the apportionment $C=\left(c_{i j}\right)$, defined through $c_{i_{1} j_{1}}:=a_{i_{1} j_{1}}+1, c_{i_{1} j_{2}}:=a_{i_{1} j_{2}}-1$ and $c_{i j}:=a_{i j}$ otherwise, we get $c_{i+}=r_{i}$ for all districts $i \leq k$ and $f_{T}(1) \leq f(C) \leq f_{T}(0)$. Analogously there exists an apportionment $C^{\prime}=\left(c_{i j}^{\prime}\right)$ with $c_{+j}^{\prime}=s_{j}$ for all parties $j \leq \ell$ and $f\left(C^{\prime}\right)=f(C)$.

Without loss of generality let $f_{T}(1)=f(C)=f\left(C^{\prime}\right)$, and thus $C \in \mathcal{A}_{T}^{r}(1)$ and $C^{\prime} \in \mathcal{A}_{T}^{s}(1)$. If $f_{T}(1)=0$, then there is nothing to be shown. Otherwise let $i \in\left(\bigcap_{A \in \mathcal{A}_{T}^{s}(0)} I^{=}(A)\right)^{c}$. With lemma 1 and equation (14) there exist two parties $j_{1}, j_{2}$ such that

$$
v_{i j_{1}}^{T}(1)=v_{i j_{1}}^{T}(0)=s\left(c_{i j_{1}}\right) \quad \text { and } \quad v_{i j_{2}}^{T}(1)=v_{i j_{2}}^{T}(0)=s\left(c_{i j_{2}}+1\right) .
$$

Analogously for any party $j \in\left(\bigcap_{A \in \mathcal{A}_{T}^{r}(0)} J^{=}(A)\right)^{c}$ there exist two districts $i_{1}^{\prime}, i_{2}^{\prime}$ such that

$$
v_{i_{1}^{\prime} j}^{T}(1)=v_{i_{1}^{\prime} j}^{T}(0)=s\left(c_{i_{1}^{\prime} j}^{\prime}\right) \quad \text { and } \quad v_{i_{2}^{\prime} j}^{T}(1)=v_{i_{2}^{\prime} j}^{T}(0)=s\left(c_{i_{2}^{\prime} j}^{\prime}+1\right) .
$$

Hence the incremental divisors are all equal to one and the AS-scaling-sequence is constant.

$\rightarrow$ (2): Let $C \in \mathcal{A}_{T}^{r}(1)$ and $i_{1}, j_{1}, j_{2}$ be defined as in the proof of part (1). With lemma 1 and equation (14) there exists a district $i_{2} \in I_{T}^{-}(0)$ such that $v_{i_{2} j_{2}}^{T}(1)=v_{i_{2} j_{2}}^{T}(0)=s\left(c_{i_{2} j_{2}}\right)$. Moreover, there exists a party $j_{3} \in J_{T}^{+}(0)$ such that $v_{i_{2} j_{3}}^{T}(1)=v_{i_{2} j_{3}}^{T}(0)=s\left(c_{i_{2} j_{3}}+1\right)$, etc. Thus, we can construct a cycle $\left(i_{1}, j_{2}\right) \rightarrow\left(i_{2}, j_{2}\right) \rightarrow \cdots \rightarrow\left(i_{q}, j_{1}\right)$ on $I_{T}(0)^{-} \times J_{T}(0)^{+}$, such that a seat-transfer is feasible. Likewise there exists a cycle on block $\left(I_{T}(0)^{-}\right)^{c} \times\left(J_{T}(0)^{+}\right)^{c}$.

By proposition 7 (1) further steps of the AS-TT-combination are well defined as long as the $L_{1}$-error does not coincide with the $L_{1}$-minimal-error. The time the $L_{1}$-error coincides with the $L_{1}$-minimal-error is called $A S$-TT-final-step. We denote it by $n_{\mathrm{AS} \text {-TT }}:=\min \left\{n \in \mathbb{N} \mid f_{T}(n)=\right.$ $\left.\lambda_{\text {min }}\right\}$. We call $\mathcal{A}_{T}(n), n=1, \ldots, n_{\mathrm{AS} \text {-TT }}$, the $A S$-TT-seat-sequence. Its $L_{1}$-error is given by $f_{T}(n):=f(A)$ for any apportionment $A \in \mathcal{A}_{T}(n)$. Given the incremental divisors

$$
\rho_{i}^{T}(n):=\left\{\begin{array}{ll}
1 & \text { if } i \in I_{T}^{-}(n-1) \\
1 / \varepsilon(n) & \text { if } i \in I_{T}^{-}(n-1)^{c}
\end{array} \quad \text { and } \quad \sigma_{j}^{T}(n):= \begin{cases}1 & \text { if } j \in J^{+}(n-1) \\
\varepsilon(n) & \text { if } i \in J_{T}^{+}(n-1)^{c},\end{cases}\right.
$$

and the respective cumulative divisors

$$
x_{i}^{T}(n):=\rho_{i}^{T}(1) \rho_{i}^{T}(2) \cdots \rho_{i}^{T}(n) \quad \text { and } \quad y_{j}^{T}(n):=\sigma_{j}^{T}(1) \cdots \sigma_{j}^{T}(n),
$$

the $A S$-TT-scaling-sequence $V_{T}(n)$ is given by

$$
v_{i j}^{T}(n)=\frac{v_{i j}^{T}(n-1)}{\rho_{i}^{T}(n) \sigma_{j}^{T}(n)}=\frac{v_{i j}^{T}(0)}{x_{i}^{T}(n) y_{j}^{T}(n)}=\frac{v_{i j}}{x_{i}\left(t_{K}\right) y_{j}\left(t_{K}\right)} \cdot \frac{1}{x_{i}^{T}(n) y_{j}^{T}(n)},
$$

where $x_{i}\left(t_{K}\right)$ and $y_{j}\left(t_{K}\right)$ denote the cumulative divisors of the AS-algorithm at time $t_{K}$. The following proposition shows that the AS-TT-final-step is reached after finitely many iterations. 


\section{Proposition 8 (AS-TT-combination).}

(1) Let the $L_{1}$-minimal-error vanish, $\lambda_{\min }=0 . \quad$ The AS-TT-combination determines the set of biproportional apportionments after finitely many steps, i.e. $n_{A S-T T}<\infty$ and $\mathcal{A}_{T}\left(n_{A S-T T}\right)=\mathcal{B}(V, r, s)$.

(2) Let the $L_{1}$-minimal-error be positive, $\lambda_{\min }>0$. The AS-TT-combination generates after finitely many steps a set of apportionments that comes with an $L_{1}$-error equal to the $L_{1^{-}}$minimal-error, i.e. $n_{A S-T T}<\infty$ and $\mathcal{A}_{T}(n)=\mathcal{B}\left(V, r, s^{\prime}\right) \cup \mathcal{B}\left(V, r^{\prime}, s\right)$ for some $r^{\prime}$ and $s^{\prime}$ such that $\sum_{i \leq k}\left|r_{i}^{\prime}-r_{i}\right|=\sum_{j \leq \ell}\left|s^{\prime}-s\right|=\lambda_{\min }$.

Proof. $\rightarrow$ (1) and (2): We show that the $L_{1}$-error decreases after at most $k+\ell$ AS-TT-steps. Let $n$ be such that $f_{T}(n)=f_{T}(n+1)>\lambda_{\min }$.

Case $\varepsilon(n+1)=\varepsilon(n+1)^{+} \geq \varepsilon(1)^{-}$: As in the proof of proposition 7 (1) there exists a cell $\left(i_{1}, j_{1}\right) \in I_{T}^{-}(0) \times J_{T}^{+}(0)^{c}$ and a matrix $C \in \mathcal{A}_{T}(n+1)$ such that $c_{i_{1} j_{1}}:=a_{i_{1} j_{1}}+1$ for all $A \in \mathcal{A}_{T}(n)$. As $f_{T}(n)=f_{T}(n+1)$ we have $j_{1} \in J_{T}^{=}(n)$. Consequently we get

$$
\begin{array}{lll}
I_{T}^{-}(n+1)=I_{T}^{-}(n) & I_{T}^{+}(n+1)=I_{T}^{+}(n) & I_{T}^{\overline{\bar{T}}(n+1)}=I_{T}^{\overline{\bar{T}}(n)} \\
J_{T}^{+}(n+1) \supsetneq J_{T}^{+}(n) & J_{T}^{-}(n+1)=J_{T}^{-}(n) & J_{T}^{=}(n+1) \subsetneq J_{T}^{=}(n) .
\end{array}
$$

Case $\varepsilon(1)=\varepsilon(1)^{-}>\varepsilon(1)^{-}$: Analogously to above we have

$$
\begin{array}{lll}
I_{T}^{-}(n+1) \supsetneq I_{T}^{-}(n) & I_{T}^{+}(n+1)=I_{T}^{+}(n) & I_{T}^{=}(n+1) \subsetneq I_{T}^{=}(n) \\
I_{T}^{+}(n+1)=I_{T}^{+}(0) & I_{T}^{-}(n+1)=I_{T}^{-}(n) & I_{T}^{=}(n+1)=I_{T}^{=}(n) .
\end{array}
$$

holds. As $\# I_{T}^{\overline{\bar{N}}}(n)+\# J_{T}^{\overline{\bar{T}}}(n)$ is bounded by $k+\ell$ the $L_{1}$-error decreases after finally many steps.

\section{Proof of proposition 4 and theorem 1 (1ii, 2ii).}

Proof of proposition 4. In case the AS-algorithm is effective the AS-algorithm determines a biproportional apportionment if and only if the flow-criterion is fulfilled, see theorem 1 . In case the AS-algorithm is ineffective the AS-TT-combination determines a biproportional apportionment if and only if the flow-criterion is fulfilled, see proposition 8.

Proof of theorem 1 (1ii, 2ii). Proposition 7 (2) and proposition 8 imply that all apportionments that are generated by the AS-TT-combination come with at least four ties.

\section{Acknowledges}

I am very grateful to my advisor Friedrich Pukelsheim. He had the privilege of advising the Zurich politicians on the amendment of their electoral law. Thus he could tell me many insights and motivated the study of the AS-algorithm. I would also like to thank my friend and colleague Fabian Reffel for his valuable remarks when finalizing the work. Moreover I thank Christoph Gietl for his remarks on earlier versions of this paper. Major parts of the paper were written during a visit with the Statistics Department of the University of California in Berkeley. The hospitality of Philip B. Stark and the support of the program 'Chancengleichheit von Frauen in Forschung und Lehre' are gratefully acknowledged. 


\section{References}

M.L. Balinski and G. Demange (1989a). An axiomatic approach to proportionality between matrices. Mathematics of Operations Research 14, 700-719.

M.L. Balinski and G. Demange (1989b). Algorithms for proportional matrices in reals and integers. Mathematical Programming 45, 193-210.

M.L. Balinski And F. Pukelsheim (2006). Matrices and politics. In: E. Liski, J. Isotalo, S. Puntanen and G.P.H. Styan (Ed.). Festschrift for Tarmo Pukkila on his 60th Birthday, 233-242. Tampere.

M.L. BAlinski AND H.P. Young (2001). Fair representation-meeting the ideal of one man, one vote (second edition). Washington DC.

M.S. BazaraA, H.D. Sherali And C.M. ShetTy (2006). Nonlinear programming: theory and algorithms (second edition). New York.

BAZI TEAM (2013). Berechnung von Anzahlen mit Zuteilungsmethoden im Internet-Calculation of allocations by apportionment methods in the internet. uni-augsburg.de/bazi.

L.M. BREgman (1967). Proof of the convergence of Sheleikhovskii's method for a problem with transportation constraints. USSR Computational Mathematics and Mathematical Physics 7, 191-204.

L.H. Cox And L.R. ERnst (1982). Controlled rounding. Information Systems and Operational Research 20, 423-432.

I. CsiszÁR (1975). I-divergence geometry of probability distributions and minimization problems. $A n$ nals of Probability 3, 146-158.

W.E. Deming And F.F. Stephan (1940). On a least squares adjustment of a sampled frequency table when the expected marginal totals are known. Annals of Mathematical Statistics 11, 427-444.

D. Gale (1957). A theorem on flows in networks. Pacific Journal of Mathematics 7, 1073-1082.

N. Gaffke And F. Pukelsheim (2008a). Divisor methods for proportional representation systems: an optimization approach to vector and matrix apportionment problems. Mathematical Social Sciences $56,166-184$.

N. Gaffke And F. Pukelsheim (2008b). Vector and matrix apportionment problems and separable convex integer optimization. Mathematical Methods of Operations Research 67, 133-159.

M. Gassner (1989). An impossibility theorem for fair bidimensional representation: towards a biproportional solution. In: E.E. Roskam (Ed.). Mathematical Psychology in Progress, 345-365. New York.

M. GASSNER (1991). Biproportional delegations: a solution for two-dimensional proportional representation. Journal of Theoretical Politics 3, 321-342.

C. Gietl and F. Reffel (2012). Accumulation points of the iterative proportional fitting procedure. To appear in Metrika.

A.J. HofFman (1960). Some recent applications of the theory of linear inequalities to extremal combinatorial analysis. Proceedings of the Symposium in Applied Mathematics of the American Mathematical Society, 113-128.

S. MAIER (2008). Biproportional apportionment methods: constraints, algorithms, and simulation. Dissertation, Augsburg University. Verlag Dr. Hut, München.

S. MAier, M. Zachariasen And P. ZaChariassen (2010). Divisor-based biproportional apportionment in electoral systems: a real-life benchmark study. Management Science 56, 373-38\%.

F. Pukelsheim (2012). An L1-analysis of the iterative proportional fitting procedure. Preprint opus.bibliothek .uni-augsburg.de/volltexte/2012/1863/.

F. Pukelsheim and C. Schuhmacher (2004). Das neue Zürcher Zuteilungsverfahren für Parlamentswahlen. Aktuelle Juristische Praxis-Pratique Juridique Actuelle 5, 505-522.

F. Pukelsheim And C. Schuhmacher (2011). Doppelproporz bei Parlamentswahlen - ein Rück- und Ausblick. Aktuelle Juristische Praxis-Pratique Juridique Actuelle 12, 1581-1599. 